\title{
Thermodynamic Modelling of Hydrogen-Multicomponent Alloy Systems: Calculating Pressure-Composition-Temperature Diagrams
}

Guilherme Zepon ${ }^{1,2,4 \#}$, Bruno Hessel Silva ${ }^{2,3}$, Claudia Zlotea ${ }^{3}$, Walter José Botta ${ }^{1,2}$, Yannick Champion ${ }^{4}$.

1. Federal University of São Carlos, Department of Materials Engineering (DEMa/UFSCar) - Rodovia Washington Luiz, km 235, São Carlos - São Paulo, Brasil CEP:13565-905.

2. Federal University of Sao Carlos, Graduate Program in Materials Science and Engineering, Federal University of São Carlos (PPGCEM/UFSCar) - Rodovia Washington Luiz, km 235, São Carlos - São Paulo, Brasil CEP:13565-905.

3. Univ Paris Est Créteil, CNRS, ICMPE, UMR 7182, 2 Rue Henri Dunant, 94320, Thiais, France.

4. Univ. Grenoble Alpes, CNRS, SIMaP, 38000 Grenoble, France.

\#corresponding author: zepon@ufscar.br

\begin{abstract}
The applicability of an alloy as a hydrogen storage media mostly relies on its pressurecomposition-temperature (PCT) diagram. Since the PCT diagram is compositiondependent, the vast compositional filed of high entropy alloys, complex concentrated alloys or multicomponent alloys can be explored to design alloys with optimized properties for each application. In this work, we present a thermodynamic model to calculate PCT diagrams of body-centered (BCC) multicomponent alloys. The entropy of the phases is described using the ideal configurational entropy for interstitial solid solutions with site blocking effect. As a first approximation, it is assumed that the $\mathrm{H}$ partial molar enthalpy of a phase is constant, so the enthalpy of $\mathrm{H}$ mixing varies linearly with the $\mathrm{H}$ concentration. Moreover, the $\mathrm{H}$ partial enthalpy of a phase for a multicomponent alloy was approximated by a simple ideal mixture law of this quantity for the alloy's components with the same structure. Experimental data and DFT calculations were used for parametrization of the enthalpy terms of eight elements ( $\mathrm{Ti}, \mathrm{V}$, $\mathrm{Cr}, \mathrm{Ni}, \mathrm{Zr}, \mathrm{Nb}, \mathrm{Hf}$, and $\mathrm{Ta}$ ), which are the components of the alloys tested in this work. Experimental PCTs of six BCC multicomponent alloys of four different systems were compared against the calculated ones and the agreement was remarkable. The model and parameters presented here can be regarded as a basis for developing powerful alloy design tools for different hydrogen storage applications.
\end{abstract}

Key words: high entropy alloys, multicomponent alloys, metal hydrides, thermodynamic model, pressure-composition-temperature. 


\section{Introduction}

Metal hydrides (MHs) are strategic materials for the widespread use of hydrogenbased energy systems. Tanks for solid-state hydrogen storage, heat-storage systems, heatpumps, hydrogen compressors, fuel cells and batteries are some of the applications in which MHs play a key role [1-6]. For each application, a different set of properties is required, and the pressure-composition-temperature (PCT) diagram of a metal-hydrogen (M-H) system usually determines its applicability. As many thermodynamic properties, the PCT diagrams can be tuned by changing the composition of the metallic material of the M-H system.

The use of alloys instead of pure metals for M-H system is not new. However, since the advent of the concepts of high entropy alloys (HEA), multi-principal element alloys (MPEA), complex concentrated alloys (CCA) or, more generally, multicomponent alloys, the vast unexplored compositional space that can be assessed for the discovery of new alloys with optimized properties was brought to light $[7,8]$. To efficiently navigate in the endless sea of compositions available, computational tools to predict phase stability and properties are paramount. Otherwise, time-consuming experimental measurements would inevitably postpone the finding of new promising materials.

In 2016, M. Shalberg et al. [9] were the first to report a body-centered cubic (BCC) HEA with promising hydrogen storage properties. The TiVZrNbHf alloy absorbed hydrogen by forming a body-centered tetragonal (BCT) hydride with high hydrogen storage capacity. The structural changes during hydrogenation of this alloy was studied in detail in [10]. In 2018, C. Zlotea et al. [11] presented the TiZrNbHfTa alloy, which also crystallized as single BCC solid solution. The authors reported that during the hydrogenation of this alloy two different hydrides could be observed. A monohydride with BCT structure stable at lower pressures and an FCC dihydride stable at higher pressures. In 2019, M. Nygard et al. [12] reported the hydrogenation of different single BCC multicomponent alloys, such as TiVNbZr, TiVNbHf, TiVNb, TiVNbTa and TiVNbCr. During hydrogenation, all these alloys formed an FCC dihydride. Moreover, an undistorted BCC monohydride was also observed for some of them, such as $\mathrm{TiVNb}$ and TiVNbCr. Such BCC monohydride was also reported for some Mg-containing HEA such as the $\mathrm{Mg}_{0.68} \mathrm{TiNbNi}_{0.55}$ alloy [13]. The understanding of the hydrides' stability at different pressures and temperatures is essential to determine the applicability of an alloy. However, PCT diagram measurements are very time-consuming and explore the 
thermodynamic properties of a large number compositions experimentally is unpractical. Therefore, the possibility of predicting the PCT diagram of an alloy based on its chemical composition would boost the search of alloys with optimized properties for each specific hydrogen storage application.

In this work we revisited the thermodynamic fundamentals of $\mathrm{M}-\mathrm{H}$ systems and present a thermodynamic model to calculate the pressure-composition-temperature (PCT) diagram of multicomponent $\mathrm{M}-\mathrm{H}$ systems. Since most of the recent works in multicomponent alloys for hydrogen storage deals with BCC alloys [9,11-17], this class of alloys was firstly considered. The enthalpy terms for eight metal elements, namely $\mathrm{Ti}$, $\mathrm{V}, \mathrm{Cr}, \mathrm{Ni}, \mathrm{Zr}, \mathrm{Nb}$, Ta, and $\mathrm{Hf}$, which are the components of the alloys considered here, were parametrized by combining experimental data and total energy calculations trough density functional theory (DFT). The calculated PCTs of six different multicomponent alloys were compared against experimental data to evaluate the prediction capability of the model, which was remarkable.

\section{Theory: Thermodynamic of M-H systems}

In multicomponent M-H systems, the complete equilibrium (CE) condition is attained when the chemical potential of all elements in the system are the same in all co-existing phases. CE is only possible when the mobility of all atoms is sufficiently high. In this case, the chemical composition of the phases is free to adjust in order to minimize the Gibbs free energy of the system. However, at the low or moderate temperatures typically employed in most hydrogen storage applications, the mobility of the metal atoms is limited. In this case, it can be assumed that only the $\mathrm{H}$ atom is mobile while the metal atoms are "frozen". In this situation, an equilibrium condition is also attained because the mobility of hydrogen allows the hydrogen chemical potential to be the same in the coexisting phases. Such condition is called para-equilibrium (PE) and it is the equilibrium condition that will be treated in this manuscript. T.B. Flanagan and W.A. Oates have already presented the main aspects of the different degrees of equilibrium that can be found in M-H systems and the readers are encouraged to refer to [18] for more details.

Let us consider a single-phase multicomponent alloy. The alloy composition is defined by the variable $c_{i}$ that is the atomic fraction of the metal element $i$. Therefore, $\sum c_{i}=1$. Since we are dealing with $\mathrm{PE}$, the composition of all possible phases in terms 
of this compositional variable will be the same. Another compositional variable $\left(c_{H}\right)$ determining the amount of hydrogen in the phase is defined as:

$$
c_{H}=\frac{n_{H}}{n_{M}}
$$

where $n_{H}$ and $n_{M}$ are the number of mols of hydrogen and metal atoms in the phase, respectively.

In this case, the Gibbs free energy $\left(\Delta G_{m}\right)$ of the possible phases in the system will depend on $c_{H}$ as shown in Figure 1 (a). The hydrogen chemical potential of $\mathrm{H}_{2}$ gas per mol of $\mathrm{H}$ (taking $P^{o}=1 \mathrm{~atm}$ as standard state) is given by equation 2, while the $\mathrm{H}$ chemical potential of the $\alpha$ - and $\beta$-phase are given by equations 3 and 4, respectively.

$$
\begin{gathered}
\mu_{H}^{H_{2}}=\frac{1}{2} R T \ln \left(\frac{P_{H_{2}}}{P^{o}}\right) \\
\mu_{H}^{\alpha}\left(c_{H}\right)=\frac{d \Delta G_{m}^{\alpha}\left(c_{H}\right)}{d c_{H}} \\
\mu_{H}^{\beta}\left(c_{H}\right)=\frac{d \Delta G_{m}^{\beta}\left(c_{H}\right)}{d c_{H}}
\end{gathered}
$$

Since the metal atoms are "frozen" and the composition of $\alpha$ - and $\beta$-phase in terms of $c_{i}$ are the same, the chemical potential of the metal atoms (M) in both phases is also a function of $c_{H}$ and is given by equations 5 and 6.

$$
\begin{array}{r}
\mu_{M}^{\alpha}\left(c_{H}\right)=\Delta G_{m}^{\alpha}\left(c_{H}\right)-c_{H} \mu_{H}^{\alpha}\left(c_{H}\right) \\
\mu_{M}^{\beta}\left(c_{H}\right)=\Delta G_{m}^{\beta}\left(c_{H}\right)-c_{H} \mu_{H}^{\beta}\left(c_{H}\right)
\end{array}
$$

It is worth noting the $\mathrm{M}$ stands for the whole set of metallic elements in the alloy and not for a specific element. Phases are in equilibrium when their chemical potential of $\mathrm{H}$ and $\mathrm{M}$ are the same. Therefore, for a given temperature, the phases in equilibrium will depend on the hydrogen pressure since $\mu_{H}^{H_{2}}$ depends on $P_{H_{2}}$. In this situation, there is only a single $P_{\mathrm{H}_{2}}$ value in which the three phases $\alpha, \beta$ and $\mathrm{H}_{2}$ can coexist in equilibrium, i.e., $\mu_{H}^{H_{2}}=\mu_{H}^{\alpha}\left(c_{H \text { plat }}^{\alpha}\right)=\mu_{H}^{\beta}\left(c_{H \text { plat }}^{\beta}\right)$ and $\mu_{M}^{\alpha}\left(c_{H \text { plat }}^{\alpha}\right)=\mu_{M}^{\beta}\left(c_{H \text { plat }}^{\beta}\right)$. The equilibrium condition is represented by the common tangent of the Gibbs free energy curves of $\alpha$ and $\beta$ phases (represented by the blue line in Figure 1 (a)). It represents the equilibrium between the $\alpha$-phase with composition $c_{H \text { plat }}^{\alpha}$, the $\beta$-phase with composition $c_{H \text { plat }}^{\beta}$ and 
the hydrogen gas at the plateau pressure $\left(P_{\text {plat }}\right)$. Figure 1 (b) presents the pressurecomposition-isotherm (PCI) diagram for the given temperature. The $P_{\text {plat }}$, which is determined by the equilibrium condition, can also be expressed by equation 7 .

$$
\frac{1}{2} R T \ln \left(\frac{P_{\text {plat }}}{P^{o}}\right)=\frac{\Delta G_{m}^{\beta}\left(c_{\text {Hplat }}^{\beta}\right)-\Delta G_{m}^{\alpha}\left(c_{\text {Hplat }}^{\alpha}\right)}{c_{\text {Hplat }}^{\beta}-c_{\text {Hplat }}^{\alpha}}
$$

Below the plateau pressure, only the $\alpha$-phase coexists in equilibrium with $\mathrm{H}_{2}$ gas and its $C_{H}$ composition will depend on the $P_{H_{2}}$. Conversely, above the plateau pressure, only the $\beta$-phase will be in equilibrium with $\mathrm{H}_{2}$ gas.

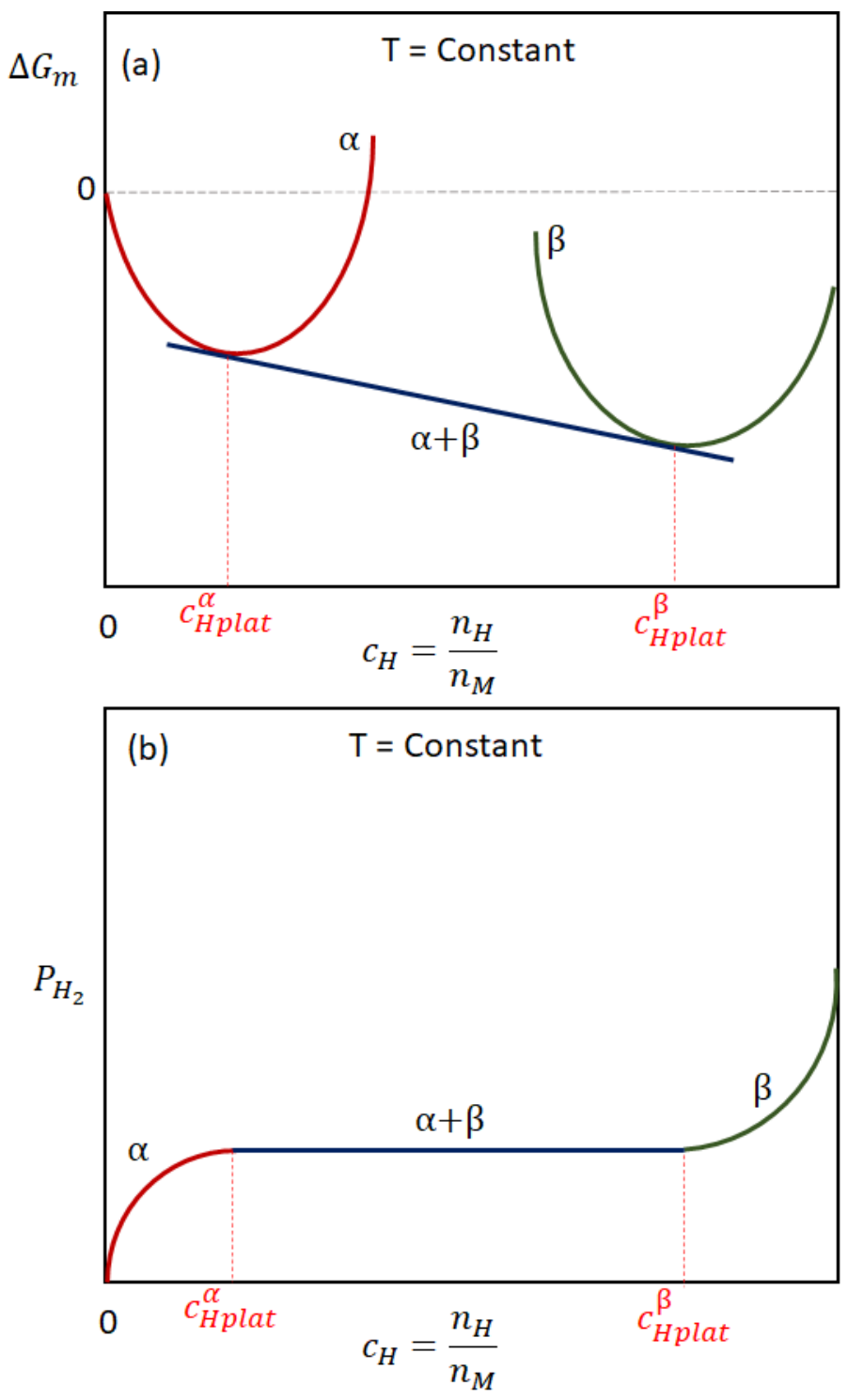

Figure 1: (a) Gibbs Free Energy of $\alpha$ and $\beta$-phase as function of $c_{H}$ for a M-H system under PE condition for a given temperature and (b) its corresponding pressurecomposition-isotherm diagram. 
It is worth noting that the Gibbs free energy of the phases depends only on $c_{H}$ because of the PE condition. Therefore, the Gibbs free energy curves presented in Figure 1 (a) can be simply understood as the Gibbs free energy of $\mathrm{H}$ mixing in the $\alpha$ - and $\beta$-phase, which is given by equation 8 .

$$
\Delta G_{m}\left(c_{H}\right)=\Delta H_{m}\left(c_{H}\right)-T \Delta S_{m}\left(c_{H}\right)
$$

$\Delta G_{m}\left(c_{H}\right)$ represents therefore the change in Gibbs free energy between a phase having composition $c_{H}$ and a reference state. For simple $\mathrm{M}-\mathrm{H}$ systems the chosen reference state is usually $\mathrm{H}_{2}$ at $1 \mathrm{~atm}$ and $\mathrm{M}$ in its stable form. In this case, since we are dealing with $\mathrm{BCC}$ alloys, it will be chosen as reference state the $\mathrm{BCC}$ alloy with $c_{H}=0$ and $\mathrm{H}_{2}$ at $1 \mathrm{~atm}$. Therefore, the enthalpy and entropy of $\mathrm{H}$ mixing are defined by equations 9 and 10 , respectively.

$$
\begin{gathered}
\Delta H_{m}\left(c_{H}\right)=H\left(M H_{C_{H}}\right)-H\left(M^{B C C}\right)-\frac{c_{H}}{2} H^{o}\left(H_{2}\right) \\
\Delta S_{m}\left(c_{H}\right)=S\left(M H_{c_{H}}\right)-S\left(M^{B C C}\right)-\frac{c_{H}}{2} S^{o}\left(H_{2}\right)
\end{gathered}
$$

where $H\left(M^{B C C}\right)$ and $S\left(M^{B C C}\right)$ are the enthalpy and entropy of the alloy in its BCC form,

$H\left(M H_{C_{H}}\right)$ and $S\left(M H_{C_{H}}\right)$ are the enthalpy and entropy of the phase ( $\alpha$ or $\beta$ ) having composition $c_{H}$, and $H^{o}\left(H_{2}\right)$ and $S^{o}\left(H_{2}\right)$ are the standard enthalpy and entropy of the $\mathrm{H}_{2}$ gas, i.e., when $P_{\mathrm{H}_{2}}=1 \mathrm{~atm}$. It is important to consider the temperature dependence of the $S^{o}\left(H_{2}\right)$, which is given by equation 11 according to [19].

$$
S_{H_{2}}^{o}=A \ln (t)+B t+\frac{C t^{2}}{2}+\frac{D t^{3}}{3}-\frac{E t^{-2}}{2}+G\left[\frac{J}{\text { mol of } H_{2}}\right]
$$

where $t=T(K) / 1000, A=33.066178, B=-11.363417, C=11.432816, D=$ $-2.772874, E=-0.158558$, and $G=172.707974$. This equation is only valid between $298 \mathrm{~K}$ and $1000 \mathrm{~K}$.

\section{Thermodynamic Model}

\subsection{Entropy of mixing}

The entropy of a phase can be expressed as the sum of the configurational $\left(S_{c}\right)$ and non-configurational $\left(S_{n c}\right)$ terms as given by equation 12 . 


$$
S=S_{c}+S_{n c}
$$

In this work, it is assumed that the difference between the non-configurational terms of $M H_{c_{H}}$ and $M$ is neglectable. Therefore, $\Delta S_{m}$ given by equation 10 becomes equation 13.

$$
\Delta S_{m}\left(c_{H}\right)=S_{c}\left(M H_{c_{H}}\right)-S_{c}(M)-\frac{c_{H}}{2} S^{o}\left(H_{2}\right)
$$

$S_{c}(M)$ is the configurational entropy of the alloy in the reference state, which will be considered as the ideal configurational entropy of a random solid solution given by equation 14 .

$$
S_{c}(M)=-R \sum_{i} c_{i} \ln c_{i}
$$

where $\mathrm{R}$ is the ideal gas constant. $S_{c}\left(M H_{C_{H}}\right)$ is the configurational entropy of the $\mathrm{H}$ solid solution or the hydride phases. $S_{C}\left(M H_{C_{H}}\right)$ is the sum of the configurational entropy of the substitutional solid solution of the metal lattice and the interstitial solid solution of $\mathrm{H}$ atoms in the interstitial sites. In this work, the configurational entropy expression proposed by J. Garcés in [20], which considers site blocking effect (SBE), will be used. When SBE takes place, the occupation of an interstitial site is prevented by the prior occupation of a neighboring site. The effective repulsion between near neighbor interstitial atoms or short-range order (SRO) between interstitial atoms may be chemical and/or strain in origin and is usually reported for M-H systems [18,21,22]. The main consequence of the SBE is to produce a dramatic decrease in the number of possible configurations, which directly affects the configurational entropy of the phase. In the model proposed by J. Garcés [20], the occupancy of one site by an interstitial atom excludes the occupancy of $(r-1)$ neighboring vacant site. Thus, the set of vacant sites is divided in two different species: $n^{f}$ free vacant sites and $n^{b}=r-1$ blocked vacant sites associated with each interstitial atom. The blocked vacant sites do not participate in the mixing process and, as consequence, it could be assumed that a new chemical specie of size $r$ is formed. For a $M H_{c_{H}}$ phase, in which only $\mathrm{H}$ atoms is considered as interstitial atoms, the expression for configurational entropy is given by equation 15 . 


$$
\begin{gathered}
S_{c}\left(M H_{c_{H}}\right)=-R\left[\sum_{i} c_{i} \ln c_{i}+c_{H} \ln \left(\frac{c_{H}}{\theta-\left[(r-1) c_{H}\right]}\right)\right. \\
\left.+\left(\theta-r c_{H}\right) \ln \left(\frac{\theta-r c_{H}}{\theta-\left[(r-1) c_{H}\right]}\right)\right]
\end{gathered}
$$

Where $\theta$ is the number of interstitial sites per metal atom. For more details regarding the deduction of this expression refers to [20]. Therefore, by replacing equation 15 and 14 into 13 , $\Delta S_{m}\left(c_{H}\right)$ becomes equation 16.

$$
\begin{aligned}
\Delta S_{m}\left(c_{H}\right)=-R & {\left[c_{H} \ln \left(\frac{c_{H}}{\theta-\left[(r-1) c_{H}\right]}\right)+\left(\theta-r c_{H}\right) \ln \left(\frac{\theta-r c_{H}}{\theta-\left[(r-1) c_{H}\right]}\right)\right] } \\
& -\frac{c_{H}}{2} S^{o}\left(H_{2}\right) \quad(16)
\end{aligned}
$$

It is worth noting that when $r=1$ no SBE is taking place. Equation 16 shows that $\Delta S_{m}$ does not depend on the configurational entropy of the metal lattice but only on the configurational entropy of the $\mathrm{H}$ interstitial solid solution. Thus, $\theta$ and $r$ are the only parameters necessary to model $\Delta S_{m}$ for each phase.

\subsection{Enthalpy of mixing}

As a first approximation, in this work we assume that the $\mathrm{H}$ partial molar enthalpy of a phase is constant for a given alloy composition. Therefore, the enthalpy of $\mathrm{H}$ mixing in a phase varies linearly with the $\mathrm{H}$ concentration as given by equation 17 .

$$
\Delta H_{m}\left(c_{H}\right)=H_{M}+h_{M} \cdot c_{H}
$$

where $H_{M}$ is a constant and $h_{M}$ is the $\mathrm{H}$ partial molar enthalpy.

\subsection{Chemical Potentials}

The thermodynamical equilibrium of the M-H system depends on the $\mu_{H}$ of the possible phases in the system. Using equations 16 and $17, \mu_{H}$ of a phase can be derived analytically resulting in equation 18 .

$$
\mu_{H}\left(c_{H}\right)=h_{M}-T\left[-R \ln \left(\frac{c_{H}\left[\theta-(r-1) c_{H}\right]^{(r-1)}}{\left[\theta-r c_{H}\right]^{r}}\right)-\frac{S_{H_{2}}^{o}}{2}\right]
$$


$\mu_{M}$ can be calculated using equation 19.

$$
\mu_{M}\left(c_{H}\right)=\Delta G_{m}\left(c_{H}\right)-c_{H} \cdot \mu_{H}\left(c_{H}\right)
$$

In possession of $\mu_{H}\left(c_{H}\right)$ and $\mu_{M}\left(c_{H}\right)$, the equilibrium of the system can be easily assessed.

\section{Parametrization}

\subsection{Phases}

Three phases are usually observed in the hydrogenation of BCC multicomponent alloys. The $\alpha$-phase is the H-dilute solid solution. In the $\alpha$-phase of BCC metals, such as $\mathrm{V}, \mathrm{Nb}$ and $\mathrm{Ta}$, the $\mathrm{H}$ atoms usually occupy the tetrahedral interstitial sites (T-sites) [23].

The $\beta$-phase is an intermediate hydride that can have different structures. For $\mathrm{Nb}$ and $\mathrm{Ta}$, the $\beta$-phase has an orthorhombic structure with the $\mathrm{H}$ atoms at some preferential $\mathrm{T}$ sites. In the case of $\mathrm{V}$, the $\beta$-phase has a monoclinic structure with $\mathrm{H}$ atoms at some preferential octahedral sites (O-sites). As already mentioned, M.M. Nygard et al. [12] reported the formation of intermediate hydrides with undistorted BCC structure for different alloys such as TiVNb and TiVCrNb. The same was observed for the $\mathrm{Mg}_{0.68} \mathrm{TiNbNi}_{0.55}$ alloy in [13]. C. Zlotea et al. [11] reported the formation of an intermediate hydride with body-centered structure (BCT) for the TiZrNbHfTa alloy. According to the authors, the BCT structure can be understood as a preferential occupancy of $\mathrm{H}$ atoms in some preferential O-sites. Regardless the crystal structure, the $\beta$-phase can be understood as an expanded (distorted or undistorted) BCC lattice with $\mathrm{H}$ atoms at some preferential interstitial site.

The $\delta$-phase is a face centered cubic structure (FCC) with $\mathrm{H}$ atoms at the T-sites in a $\mathrm{CaF}_{2}$-type structure. This phase is usually observed during hydrogenation of pure elements such as $\mathrm{VH}_{2}, \mathrm{NbH}_{2}, \mathrm{TiH}_{2}$, and for several multicomponent BCC alloys such as TiVNb, TiVNbCr and TiNbZrHfTa $[12,13]$. $\delta$-phase with BCT structure has also been reported for the TiVZrNbHf alloy $[9,17]$. The BCT structure is only a slight distortion of the FCC structure. The formation of such distorted $\delta$-phase during hydrogenation has been reported for some pure metals and other alloys as well. This is the case of $\mathrm{ZrH}_{2}$, $\mathrm{HfH}_{2},(\mathrm{TiZr}) \mathrm{H}_{\mathrm{x}}$, and (TiHfZr) $\mathrm{H}_{\mathrm{x}}$ hydrides [24-26]. 


\subsection{Entropy of mixing}

For the entropy of mixing of each phase it is necessary only to define the values of $\theta$ and $r$ parameters of equation 16.

In this work, we will assume that for the $\alpha$-phase the $H$ atoms occupy the T-sites of the $\mathrm{BCC}$ structure, therefore, $\theta=6$. In the $\mathrm{BCC}$ structure, a tetrahedral site has four first T-site neighbors, two second T-site neighbors and eight third T-site neighbors at distances of $a \sqrt{2} / 4, a / 2$ and $a \sqrt{6} / 4$, respectively. Thus, $r=5$ if only the first T-site neighbors are blocked by the prior occupation of a T-site. If first and second neighbors are blocked, then $r=7$. And if the first, second and third neighbors are blocked, $r=15$.

For the $\beta$-phase, the $\mathrm{H}$ atoms can occupy either some preferential T-sites or O-sites. For the cases in which T-sites are occupied, we will assume that only a fraction of them is initially available (ordered structure). In this case we chose $\theta=4$ (an intermediate value between the $\alpha$ - and $\delta$-phase). When the $\mathrm{O}$-sites are occupied, $\theta=3$. If we consider that only one $\mathrm{T}$ - or $\mathrm{O}$-site is blocked by the prior occupation of a T- or O-site, $r=2$. If two sites are blocked, then $r=3$.

In the $\delta$-phase, the $\mathrm{H}$ atoms occupy the T-sites of the FCC structure, therefore, $\theta=2$. No SBE is considered to occur in the $\delta$-phase, therefore, $r=1$. Table 1 summarizes $\theta$ and $r$ values that can be used for each phase.

Table 1: Possible $\theta$ and $r$ entropy parameters for the different phases.

\begin{tabular}{|c|c|c|}
\hline & $\boldsymbol{\theta}$ & $\boldsymbol{r}$ \\
\hline $\boldsymbol{\alpha}$-phase & 6 & 5,7 or 15 \\
\hline $\boldsymbol{\beta}$-phase & 4 or 3 & 3 or 2 \\
\hline $\boldsymbol{\delta}$-phase & 2 & 1 \\
\hline
\end{tabular}

\subsection{Enthalpy of mixing}

\subsubsection{Total Energy Calculation by DFT}

For the parametrization of the $H_{M}$ and $h_{M}$ enthalpy terms, the total energy of different structures was calculated using first principles calculations in the framework of the 
density functional theory (DFT). The calculations were carried out using the WIEN2k program, which is based on a full-potential (considers all electrons - core and valence) augmented plane wave plus local orbitals $(A P W+10)$ method to solve the Kohn-Sham equations of DFT [27,28]. The exchange and correlation energy were treated using the generalized gradient approximation (GGA) parametrized by Perdew, Burke and Ernzerhof (PBE) [29]. The cut-off energy is defined as RMT*Kmax, where RMT is the smallest atomic sphere radius in the unit cell and Kmax is the largest k-vector in the planewave expansion. In this work, the structures containing $\mathrm{H}$ atoms were calculated using $\mathrm{RMT}^{*} \mathrm{Kmax}=4.0$. The calculation of the structures having only metal atoms was carried out using RMT*Kmax $=7.5$. For all calculations, $10.000 \mathrm{k}$-points were used in the irreducible part of the Brillouin zone. The energy convergence criteria for the selfconsistent field calculation was $0.0001 \mathrm{Ry}$. The total ground state energy and the equilibrium volume of the structures at $0 \mathrm{~K}$ were found by calculating the total energy of the structures with different lattice parameters and fitting the results with the Murnaghan equation of state.

\subsubsection{Parameterization of $\boldsymbol{H}_{M}$}

As the BCC metal or alloy with $c_{H}=0$ is taken as reference state, $H_{M}^{\alpha}=0$. Since the $\beta$-phase is an expanded and un/distorted BCC phase, we can assume that when $c_{H} \rightarrow 0$, the $\alpha$ - and $\beta$-phase are practically the same, therefore, $H_{M}^{\beta}=0$.

In this work, the $\delta$-phase will be treated only as an FCC phase and possible distortions

will be neglected. In this case, $H_{M}^{\delta}$ represents the enthalpy of phase transition from the metal or alloy in the reference state (BCC) to the FCC structure. For the pure metals, we can approximate this value by simply calculating the difference between the total energies of the FCC and BCC structure. For multicomponent alloys, we consider that the total energy of random solid solution is divided among the M-M bonds between the first neighbor atoms. The number of bonds considered per metal is therefore equal to the coordination number of the structure ( 8 for $\mathrm{BCC}$ and 12 for FCC) divided by two. In this case, the total energy of $\alpha$-phase and $\delta$-phase when $c_{H}=0$ is given by equations 20 and 21 , respectively, and $H_{M}^{\delta}$ is given by equation 22 .

$$
E_{\text {total }}^{\alpha}=4 \varepsilon_{M-M}^{\alpha}
$$




$$
\begin{gathered}
E_{\text {total }}^{\delta}=6 \varepsilon_{M-M}^{\delta} \\
H_{M}^{\delta}=E_{\text {total }}^{\delta}-E_{\text {total }}^{\alpha}
\end{gathered}
$$

where $\varepsilon_{M-M}^{\alpha}$ and $\varepsilon_{M-M}^{\delta}$ is the bond energy of the first neighbor atoms of $\alpha$ - and $\delta$ phase, respectively. In this work, it will be assumed that for a multicomponent alloy $\varepsilon_{M-M}^{\alpha}$ and $\varepsilon_{M-M}^{\delta}$ assumes an average value of all the existing bond types in the structure weighted by their probability of appearance as given by equation 23 .

$$
\varepsilon_{M-M}=\sum_{i, j} \delta_{i j} \cdot p_{i j} \cdot \varepsilon_{i-j}
$$

where $\varepsilon_{i-j}$ is the energy of a bond between element $i$ and $j$; $p_{i j}$ is the probability of appearance of the bond $i$-j, which for a random solid solution is simply given by $c_{i} \cdot c_{j}$; and $\delta_{i j}$ is the degeneracy of the bond $i-j$, which is 1 for $i=j$ and 2 for $i \neq j$.

To calculate the energy values of $(i-i)$-type bonds $\left(\varepsilon_{i-i}\right)$, the total energies of the BCC and FCC structures of metal $i$ were calculated by DFT and the values of $\varepsilon_{i-i}^{\alpha}$ and $\varepsilon_{i-i}^{\delta}$ were determined using equation 20 and 21 , respectively. To find the values of $\varepsilon_{i-j}^{\alpha}$ (i.e. $i \neq j$ ), the total energies of B2 structures (as shown in Figure 2 (a)) were calculated and equation 23 applied. To find $\varepsilon_{i-j}^{\delta}$, the total energies of $\mathrm{L} 1_{0}$ structures with c/a $=1$ (Figure 2 (b)) were calculated. In this case, the value of the $\varepsilon_{i-j}^{\delta}$ is found using equation 24.

$$
E_{\text {total }}^{L 1_{0}}=4 \varepsilon_{i-j}^{\delta}+\varepsilon_{i-i}^{\delta}+\varepsilon_{j-j}^{\delta}
$$

Table 2 shows the values of $\varepsilon_{i-j}^{\alpha}$ and $\varepsilon_{i-j}^{\delta}$ and Figure S1 of the supplementary data file shows the $\varepsilon_{i-j} v s$ bond length curves for the atomic pairs considered in this work. It is worth stressing that we choose to use the minimum values of $\varepsilon_{i-j}$. In this case, it is assumed that all the bonds are in the equilibrium distance for each atomic pair. Therefore, the total energy calculated using equations 20, 21 and 24 can be regarded as a lower boundary energy. It is important to bear in mind that this model intends only to find reasonable values for $H_{M}^{\delta}$ and not an accurate description of the total energies and enthalpies of multicomponent alloys, for which higher order atomic interactions and temperature might have important effects. 


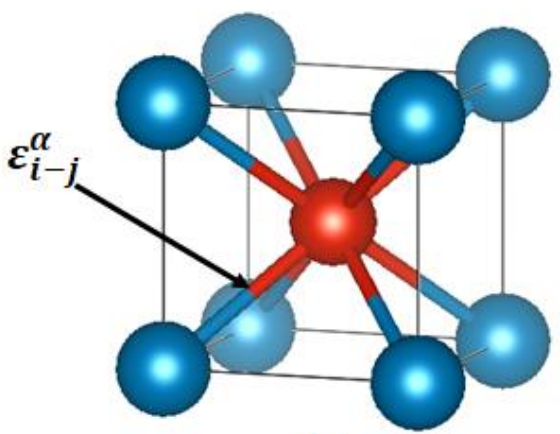

(a)

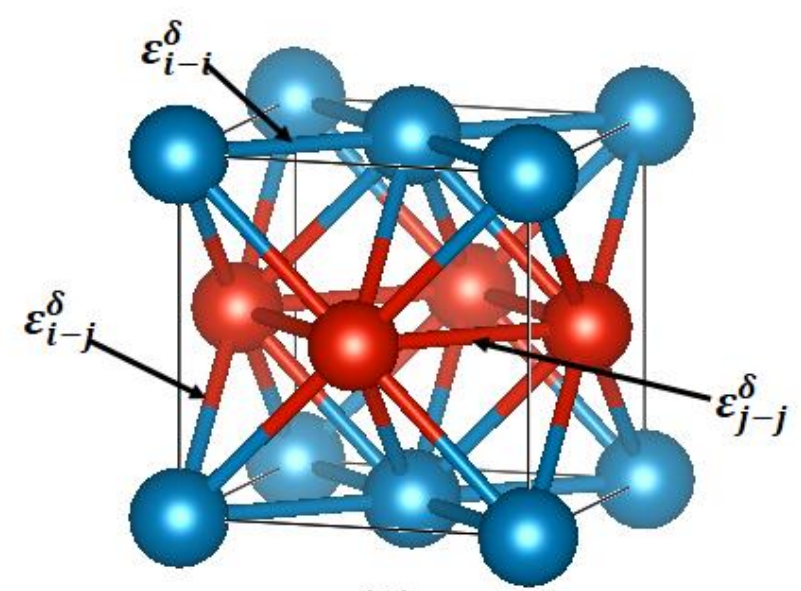

(b)

Figure 2: (a) B2 unit cell having 8 bonds with energy $\varepsilon_{i-j}^{\alpha}$.(b) L1 $1_{0}$ unit cell having 16 bonds with energy $\varepsilon_{i-j}^{\delta}, 4$ bonds with energy $\varepsilon_{i-i}^{\delta}$ and 4 bonds having energy $\varepsilon_{j-j}^{\delta}$. 
Table 2: $\varepsilon_{i-j}^{\alpha}$ and $\varepsilon_{i-j}^{\delta}$ bond energies in $\mathrm{kJ} / \mathrm{mol}$ for the atomic pairs considered in this work.

\begin{tabular}{|c|c|c|c|c|c|c|c|c|c|}
\hline$\varepsilon_{i-j}^{\alpha}$ & $\mathbf{T i}$ & $\mathbf{V}$ & $\mathrm{Cr}$ & $\mathbf{N i}$ & $\mathbf{Z r}$ & $\mathbf{N b}$ & Hf & $\mathbf{T a}$ & $\varepsilon_{i-j}^{\alpha}$ \\
\hline & -560421.1 & -591764.2 & -625097.6 & -779338.3 & -1461425.9 & -1534040.5 & -5235125.7 & -5408502.9 & $\mathbf{T i}$ \\
\hline$\varepsilon_{i-j}^{\delta}$ & & -623110.6 & -656445.6 & -810672.3 & -1492766.4 & -1565384.6 & -5266468.0 & -5439848.9 & $\mathbf{V}$ \\
\hline $\mathbf{T i}$ & -373615.0 & & -689776.6 & -843996.9 & -1526098.3 & -1598717.9 & -5299801.2 & -5473183.0 & $\mathrm{Cr}$ \\
\hline $\mathbf{V}$ & -394508.8 & -415403.3 & & -998231.4 & -1680343.3 & -1752948.9 & -5454044.2 & -5627411.5 & $\mathbf{N i}$ \\
\hline $\mathrm{Cr}$ & -416729.4 & -437623.9 & -459844.9 & & -2362431.4 & -2435044.2 & -6136130.7 & -6309505.1 & $\mathbf{Z r}$ \\
\hline $\mathbf{N i}$ & -519564.3 & -540455.6 & -562671.0 & -665488.5 & & -2507661.1 & -6208744.5 & -6382124.0 & $\mathbf{N b}$ \\
\hline $\mathbf{Z r}$ & -974285.1 & -995177.5 & -1017397.7 & -1120235.8 & -1574955.1 & & -9909830.0 & -10083205.7 & Hf \\
\hline $\mathbf{N b}$ & -1022693.3 & -1043586.6 & -1065807.2 & -1168642.9 & -1623361.7 & -1671769.0 & & -10256586.5 & Ta \\
\hline Hf & -3490085.2 & -3510978.4 & -3533199.2 & -3636036.0 & -4090755.0 & -4139162.4 & -6606555.1 & & $\varepsilon_{i-j}^{\alpha}$ \\
\hline Ta & -3605668.2 & -3626562.9 & -3648783.8 & -3751617.8 & -4206336.2 & -4254744.8 & -6722137.1 & -6837720.5 & \\
\hline$\overline{\varepsilon_{i-j}}$ & $\mathbf{T i}$ & $\mathbf{V}$ & $\mathrm{Cr}$ & $\mathbf{N i}$ & $\mathbf{Z r}$ & $\mathbf{N b}$ & Hf & Ta & $\varepsilon_{i-j}^{\delta}$ \\
\hline
\end{tabular}




\subsubsection{Parametrization of $h_{M}$}

$h_{M}$ represents the $\mathrm{H}$ partial molar enthalpy of the phase. As a first treatment, we will assume that the $h_{M}$ of a multicomponent phase will be approximately the mean value of the $\mathrm{H}$ partial molar enthalpy of the pure elements in the same phase, as given by equation 25.

$$
h_{M}=\sum_{i} c_{i} \cdot h_{i}
$$

where $c_{i}$ and $h_{i}$ are the atomic fraction and $\mathrm{H}$ partial molar enthalpy for element $i$ in the phase, respectively.

For $h_{i}^{\alpha}$ and $h_{i}^{\beta}$ we used the experimental values of enthalpy of $\mathrm{H}$ solution at infinite dilution $\left(\Delta H_{\infty}\right)$ of the elements. These values were chosen based on two observations described by Y. Fukai [21]. First, for elements existing in different allotropic forms, $\Delta H_{\infty}$ are approximately the same regardless the allotropic form. Second, for BCC metals, the standard enthalpy of formation $\left(\Delta H_{f}^{o}\right)$ of the intermediate hydrides ( $\beta$-phase) are often remarkably close to $\Delta H_{\infty}$ for the respective metals. This is true even for $\mathrm{V}$, in which $\mathrm{H}$ atoms occupy the T-sites in the $\alpha$-phase and the O-sites in the highly distorted $\beta$-phase. According to Y. Fukai [21], the enthalpy of H solution in these phases is more related to the electronic structure of the host metal than its crystal structure or the hydrogen content. The values of $\Delta H_{\infty}$ for the eight elements considered in this work were taken from the compilation made by R. Griessen and T. Riesterer in [30]. Table 3 presents the values of $h_{i}^{\alpha}$ and $h_{i}^{\beta}$ for the eight elements.

For the $\delta$-phase, the values of $h_{i}^{\delta}$ was determined using DFT calculation. The total energy of $\delta-\mathrm{MH}_{2}$ hydrides and $\delta-\mathrm{M}$ metal (i.e. $\mathrm{M}$ with FCC structure) was calculated and $h_{i}^{\delta}$ was determined by equation 26 :

$$
h_{i}^{\delta}=\frac{E_{\text {total }}^{\delta-M H_{2}}-E_{\text {total }}^{\delta-M}-E_{\text {total }}^{H_{2}}}{2}
$$

where $E_{\text {total }}^{\mathrm{H}_{2}}$ is the total energy of the $\mathrm{H}_{2}$ gas, which was determined using the experimental data of $\Delta H_{f}^{o}$ for $\delta-\mathrm{VH}_{2}, \delta-\mathrm{TiH}_{2}$ and $\delta-\mathrm{NbH}_{2} . \Delta H_{f}^{o}$ can be approximated using equation 27. 


$$
\Delta H_{f}^{o}\left(M H_{2}\right)=E_{\text {total }}^{\delta-M H_{2}}-E_{\text {total }}^{M}-E_{\text {total }}^{H_{2}}
$$

where $E_{\text {total }}^{M}$ is the total energy of $\mathrm{M}$ in its standard state, which is BCC for $\mathrm{V}$ and $\mathrm{Nb}$ and HCP for Ti. Table 4 summarizes the results and Figure S2 of the supplementary data file shows the $E_{\text {total }} v s$ unit cell volume curves for the calculated structures. The mean value of $E_{\text {total }}^{\mathrm{H}_{2}}=-3073.3 \mathrm{~kJ} / \mathrm{mol}$ was used to calculate $h_{i}^{\delta}$ using equation 26 . Table 3 also presents the calculated values of $h_{i}^{\delta}$ for the eight elements considered in this work. Figure $\mathrm{S} 3$ of the supplementary data file shows the $E_{\text {total }} v s$ unit cell volume curves for the calculated structures.

Table 3: Values of $h_{i}^{\alpha}, h_{i}^{\beta}$ and $h_{i}^{\delta}$ for the elements considered in this work. Values in $\mathrm{kJ} / \mathrm{mol}$ of $\mathrm{M}$.

\begin{tabular}{|c|c|c|}
\hline Element $\boldsymbol{i}$ & $\boldsymbol{h}_{\boldsymbol{i}}^{\boldsymbol{\alpha}}=\boldsymbol{h}_{\boldsymbol{i}}^{\boldsymbol{\beta}}$ & $\boldsymbol{h}_{\boldsymbol{i}}^{\boldsymbol{\delta}}$ \\
\hline $\mathbf{T i}$ & -58.2 & -64.2 \\
\hline $\mathbf{V}$ & -29.5 & -31.8 \\
\hline $\mathbf{C r}$ & +52.0 & 2.2 \\
\hline $\mathbf{N i}$ & +16.0 & 18.5 \\
\hline $\mathbf{Z r}$ & -64.0 & -74.4 \\
\hline $\mathbf{N b}$ & -35.3 & -40.8 \\
\hline $\mathbf{H f}$ & -38.0 & -52.0 \\
\hline $\mathbf{T a}$ & -36.0 & -6.4 \\
\hline
\end{tabular}

Table 4: Experimental value of $\Delta H_{f}^{o}\left(\delta-M H_{2}\right)$ and calculated values of $E_{\text {total }}^{\delta-M H_{2}}, E_{\text {total }}^{M}$ and $E_{\text {total }}^{\mathrm{H}_{2}}$ for $\mathrm{M}=\mathrm{V}$, Ti and $\mathrm{Nb}$. All values are given in $\mathrm{kJ} / \mathrm{mol}$ of $\mathrm{M}$ or $\mathrm{H}_{2}$.

\begin{tabular}{|c|c|c|c|c|}
\hline $\boldsymbol{M}$ & $\Delta \boldsymbol{H}_{\boldsymbol{f}}^{\boldsymbol{o}}[\mathrm{Ref}]$ & $\boldsymbol{E}_{\text {total }}^{\boldsymbol{\delta}-\mathbf{M H}_{\mathbf{2}}}$ & $\boldsymbol{E}_{\text {total }}^{\boldsymbol{M}}$ & $\boldsymbol{E}_{\text {total }}^{\boldsymbol{H}_{\mathbf{2}}}$ \\
\hline $\mathbf{V}$ & $-40.6[21]$ & -2495556.7 & -2492442.5 & -3073.6 \\
\hline $\mathbf{T i}$ & $-134.0[30]$ & -2244891.6 & -2241695.3 & -3062.3 \\
\hline $\mathbf{N b}$ & $-40.6[21]$ & -10033769.0 & -10030644.6 & -3083.9 \\
\hline
\end{tabular}




\section{Materials and Methods}

\subsection{PCT Calculation}

In this work, we wrote a simple code in Microsoft Excel using the VBA and solver tools to find the equilibrium conditions of the systems. This was done by varying $c_{H}$ of the phases and minimizing the differences between $\mu_{H}$ and $\mu_{M}$ of them, i.e., finding the common tangents of the Gibbs free energy curves. To do so, the Gibbs free energy of the phases should be described in all $c_{H}$ range. Because the natural logarithm in the configurational entropy expression, this quantity can be calculated using equation 16 only up to $c_{H}=\theta / r$. To solve this problem, we fitted the configurational entropy curves with a sixth order polynomial equation. For $c_{H} \geq \theta / r$ the polynomial equations were used to describe the configurational entropy. Figure S4 of the supplementary data file shows the fitting for the different possible values of $\theta$ and $r$ presented in Table 1.

\subsection{Materials and experimental PCT measurement.}

Samples of four multicomponent alloys, namely ( $\mathrm{TiVCr})_{85} \mathrm{Cr}_{15}, \mathrm{Ti}_{28.3} \mathrm{~V}_{1.3} \mathrm{Nb}_{55.4} \mathrm{Cr}_{15}$, $(\mathrm{TiVNb})_{96.8} \mathrm{Ni}_{3.2}$ and $\mathrm{Ti}_{32.1} \mathrm{~V}_{36.1} \mathrm{Nb} 28 \mathrm{Ni}_{3.8}$, were produced by arc-melting. Characterization trough X-ray diffraction (XRD), scanning electron microscopy (SEM) and energy dispersive X-ray spectroscopy (EDX) showed that the ( $\mathrm{TiVCr})_{85} \mathrm{Cr}_{15}$ and $\mathrm{Ti}_{28.2} \mathrm{~V}_{1.3} \mathrm{Nb}_{55.4} \mathrm{Cr}_{15}$ were single $\mathrm{BCC}$ solid solutions with high chemical homogeneity. On the other hand, the $(\mathrm{TiVNb})_{96.8} \mathrm{Ni}_{3.2}$ and $\mathrm{Ti}_{32.1} \mathrm{~V}_{36.1} \mathrm{Nb}_{28} \mathrm{Ni}_{3.8}$ presented a microstructure composed of a major BCC matrix and a small fraction of Ni-rich intermetallic. The chemical composition of the BCC matrixes was determined through SEM-EDX analyses, resulting in $\mathrm{Ti}_{31.7} \mathrm{~V}_{32.4} \mathrm{Nb}_{33.7} \mathrm{Ni}_{2.2}$ for the (TiVNb) ${ }_{96.8} \mathrm{Ni}_{3.8}$ alloy and $\mathrm{Ti}_{30.1} \mathrm{~V}_{35.5} \mathrm{Nb}_{32.2} \mathrm{Ni}_{2.2}$ for the $\mathrm{Ti}_{32.1} \mathrm{~V}_{36.1} \mathrm{Nb}_{28} \mathrm{Ni}_{3.8}$ alloy. The compositions of the BCC matrixes were used to calculate the PCT diagrams.

PCI measurements were carried out in a Sieverts-type apparatus. Small sample pieces (cut using a manual shear cutter) were used for measurements. Initially, the samples were subjected to an activation procedure at $390{ }^{\circ} \mathrm{C}$ under dynamic vacuum for 12 hours to facilitate hydrogen absorption. PCI measurements at three different temperatures at least were obtained in absorption and desorption conditions applying variable doses of 
hydrogen up to the maximum pressure of 100 bar. The low-pressure limit of the pressure gauge was $10^{-3} \mathrm{~atm}$.

\section{Results}

In addition to the four alloys presented in section 5.2, two alloys whose PCT measurements were reported in literature, namely, TiZrNbHfTa [11] and TiVZrNbHf [17] were also analyzed. Table 5 shows the $\theta$ and $r$ parameters used to describe configurational entropy as well as the calculated values of $H_{M}$ and $h_{M}$ of the three phases for all studied alloys. The same values of $\theta$ and $r$ were used for the $\alpha$ - and $\delta$-phase for the six alloys. For the $\alpha$-phase, it was considered the blocking of first and second T-site neighbors. However, due to the possible differences in the nature of the $\beta$-phase for each alloy, the values of $\theta$ and $r$ for the $\beta$-phase were adjusted to better describe the experimental results.

Table 5: $\theta$ and $r$ parameters used to describe the configurational entropy and the calculated values of $H_{M}$ and $h_{M}$ to describe the enthalpy of mixing of the three phases for each alloy. The values of $H_{M}$ and $h_{M}$ are in $\mathrm{kJ} / \mathrm{mol} . H_{M}^{\alpha}=H_{M}^{\beta}=0$.

\begin{tabular}{|c|c|c|c|c|c|c|c|c|c|c|}
\hline \multirow{2}{*}{ Alloy } & \multicolumn{3}{|c|}{$\alpha$-phase } & \multicolumn{3}{c|}{$\beta$-phase } & \multicolumn{4}{c|}{$\delta$-phase } \\
\cline { 2 - 11 } & $\boldsymbol{\theta}$ & $\boldsymbol{r}$ & $\boldsymbol{h}_{\boldsymbol{M}}$ & $\boldsymbol{\theta}$ & $\boldsymbol{r}$ & $\boldsymbol{h}_{\boldsymbol{M}}$ & $\boldsymbol{\theta}$ & $\boldsymbol{r}$ & $\boldsymbol{H}_{\boldsymbol{M}}$ & $\boldsymbol{h}_{\boldsymbol{M}}$ \\
\hline${\text { (TiVCr) }{ }_{\mathbf{2}} \mathbf{C r}_{15}}^{6}$ & 7 & -27 & 4 & 2 & -27 & 2 & 1 & +15.6 & -38.4 \\
\hline Ti28.3V1.3Nb55.4Cr15 & 6 & 7 & -28.6 & 4 & 2 & -28.6 & 2 & 1 & +16.9 & -40.9 \\
\hline Ti31.7V32.4Nb33.7Ni2.2 & 6 & 7 & -39.5 & 4 & 3 & -39.5 & 2 & 1 & +8.7 & -44.0 \\
\hline Ti30.1V35.5Nb32.2Ni2.2 & 6 & 7 & -38.9 & 4 & 3 & -38.9 & 2 & 1 & +9.1 & -43.3 \\
\hline TiZrNbHfTa & 6 & 7 & -46.3 & 3 & 2 & -46.3 & 2 & 1 & +3.2 & -47.6 \\
\hline TiVZrNbHf & 6 & 7 & -45 & 4 & 2 & -45 & 2 & 1 & +2.5 & -52.6 \\
\hline
\end{tabular}

Figure 3 presents the comparison of the calculated and experimental PCTs for the six alloys. All the calculated Gibbs free energy curves as well as the common tangents that determine the equilibrium plateaus are presented in Figures S5 to S10 of the supplementary data file. The calculated PCT of the (TiVCr) ${ }_{85} \mathrm{Cr}_{15}, \mathrm{Ti}_{28.3} \mathrm{~V}_{1.3} \mathrm{Nb}_{55.4} \mathrm{Cr}_{15}$, $\mathrm{Ti}_{31.7} \mathrm{~V}_{32.4} \mathrm{Nb}_{33.7} \mathrm{Ni}_{2.2}$ and $\mathrm{Ti}_{30.1} \mathrm{~V}_{35.5} \mathrm{Nb}_{32.2} \mathrm{Ni}_{2.2}$ alloys presented two plateaus. The first plateau at low hydrogen concentration between the $\alpha$ - and $\beta$-phase, and a second plateau 
at higher hydrogen concentration between the $\beta$ - and $\delta$-phase. The experimental results confirmed the presence of two plateaus. However, only the second plateau could be experimentally determined. XRD after partial absorption at room temperature confirmed that in all cases the $\beta$-phase was an expanded and undistorted BCC phase. We suppose that when the $\beta$-phase is undistorted, the $\mathrm{H}$ atoms continues to occupy the $\mathrm{T}$-sites and for this reason we chose $\theta=4$ to describe the configurational entropy of this phase. The experimental results show that hysteresis of approximately one order of magnitude takes place in the second plateau for all the alloys. For the ( $\mathrm{TiVCr})_{85} \mathrm{Cr}_{15}$ alloy, the calculated PCIs were in between the plateaus measured in absorption and desorption. On the other hand, for the $\mathrm{Ti}_{28.3} \mathrm{~V}_{1.3} \mathrm{Nb}_{55.4} \mathrm{Cr}_{15}, \mathrm{Ti}_{31.7} \mathrm{~V}_{32.4} \mathrm{Nb}_{33.7} \mathrm{Ni}_{2.2}$ and $\mathrm{Ti}_{30.1} \mathrm{~V}_{35.5} \mathrm{Nb}_{32.2} \mathrm{Ni}_{2.2}$ alloys, the calculated second plateaus were close to the experimental desorption plateau. It is worth noting that for the four compositions, the calculated values of $c_{H p l a t}^{\beta}$ in the second plateau reasonably agree with the experimental ones. On the other hand, the calculated values of $c_{\text {Hplat }}^{\delta}$ are higher than the experimental ones. The model predicts that $c_{\text {Hplat }}^{\delta}$ decreases with the increase of temperature, however, the decreasing in the calculated values of $c_{\text {Hplat }}^{\delta}$ are lower than those observed experimentally. This phenomenon still needs further investigation. Despite this, one can see that for the four alloys the agreement between the calculated plateau pressures with the experimental results was remarkable.

Table 6 presents the calculated values of plateau enthalpy $\left(\Delta H_{\text {plat }}\right)$ and plateau entropy $\left(\Delta S_{\text {plat }}\right)$, which are defined by equation 28 and 29 , respectively.

$$
\begin{aligned}
\Delta H_{\text {plat }} & =\frac{\Delta H_{m}^{\delta}\left(c_{\text {Hplat }}^{\delta}\right)-\Delta H_{m}^{\beta}\left(c_{\text {Hplat }}^{\beta}\right)}{c_{\text {Hplat }}^{\delta}-c_{\text {Hplat }}^{\alpha}} \\
\Delta S_{\text {plat }} & =\frac{\Delta S_{m}^{\delta}\left(c_{\text {Hplat }}^{\delta}\right)-\Delta S_{m}^{\beta}\left(c_{\text {Hplat }}^{\beta}\right)}{c_{\text {Hplat }}^{\delta}-c_{\text {Hplat }}^{\beta}}
\end{aligned}
$$

Experimentally, $\Delta H_{\text {plat }}$ and $\Delta s_{\text {plat }}$ are estimated using the so called Van't Hoff relationship (equation 30).

$$
\frac{1}{2} \ln \left(P_{\text {plat }}\right)=\frac{\Delta H_{\text {plat }}}{R T}-\frac{\Delta S_{\text {plat }}}{R}
$$

In this case, it is assumed that $\Delta H_{\text {plat }}$ and $\Delta s_{\text {plat }}$ are constant over temperature and, therefore, these quantities can be found directly by the linearization of $\frac{1}{2} \ln \left(P_{\text {plat }}\right)$ vs $1 / T$. 
However, neither $\Delta H_{\text {plat }}$ nor $\Delta S_{\text {plat }}$ is actually constant over temperature since the phase boundaries, i.e. $c_{H \text { plat }}^{\beta}$ and $c_{\text {Hplat }}^{\delta}$, varies with temperature. Moreover, since $S_{H_{2}}^{o}$ varies with temperature (equation 11), $\Delta S_{\text {plat }}$ would not be constant even if $c_{\text {Hplat }}^{\beta}$ and $c_{\text {Hplat }}^{\delta}$ were. Because of the variation of $\Delta H_{\text {plat }}$ and $\Delta S_{\text {plat }}$ with temperature is often small, the value obtained from the linearization of equation 25 is often a good approximation of the actual values. Table 6 also shows the values of $\Delta H_{\text {plat }}$ and $\Delta S_{\text {plat }}$ determined experimentally. Figure 4 presents a Van't Hoff plot comparing the calculated and experimental values of the plateau pressure over temperature. Tables S1 to S6 of the supplementary data file presents all the calculated plateau properties for the six studied alloys.

One can see that the agreement between the calculated and experimental values of $\Delta H_{\text {plat }}$ for the $(\mathrm{TiVCr})_{85} \mathrm{Cr}_{15}$ and $\mathrm{Ti}_{28.3} \mathrm{~V}_{1.3} \mathrm{Nb}_{55.4} \mathrm{Cr}_{15}$ are also remarkable. Moreover, the experimental $\Delta S_{\text {plat }}$ lies between the values of calculated at $25^{\circ} \mathrm{C}$ and $200{ }^{\circ} \mathrm{C}$, also showing a good agreement. For the $\operatorname{Ti}_{31.7} \mathrm{~V}_{32.4} \mathrm{Nb}_{33.7} \mathrm{Ni}_{2.2}$, the calculated $\Delta H_{\text {plat }}$ were slightly overestimated ( -37 to $-38 \mathrm{~kJ} / \mathrm{mol}$ against -32 to $-35 \mathrm{~kJ} / \mathrm{mol}$ ) and the experimental and calculated values of $\Delta S_{\text {plat }}$ were in good agreement. For the $\mathrm{Ti}_{30.1} \mathrm{~V}_{35.5} \mathrm{Nb}_{32.2} \mathrm{Ni}_{2.2}$ alloy, the experimental values of $\Delta H_{\text {plat }}$ had a larger variation between absorption and desorption measurements (-34.2 and $-38.1 \mathrm{~kJ} / \mathrm{mol}$, respectively), which comprises the calculated values. In this case, the experimental values of $\Delta S_{\text {plat }}$ was slightly higher than the calculated ones.

Figure 3 (e) shows the calculated PCT for the TiZrNbHfTa alloy as well as its experimental absorption PCI at $300^{\circ} \mathrm{C}$ taken from [11]. C. Zlotea et al. [11] suggested that the $\beta$-phase for this alloys is BCT with the $\mathrm{H}$ at $\mathrm{O}$-sites. For this reason, we chose $\theta$ $=3$ and $r=2$ to describe the configurational entropy of this phase. The calculated PCT also presented a first plateau between the $\alpha$ - and $\beta$-phase and a second plateau between the $\beta$ - and $\delta$-phase. One can see that at $300{ }^{\circ} \mathrm{C}$ the experimental absorption of the second plateau pressure is about $20 \mathrm{~atm}$, whereas the calculated one is $2 \mathrm{~atm}$. Considering the one order of magnitude hysteresis observed for the other BCC alloys, we believe that the calculated value of $P_{\text {plat }}$ is probably close to the actual one. The calculated values of $\Delta H_{\text {plat }}$ and $\Delta S_{\text {plat }}$ for the TiZrNbHfTa alloys are presented in Table 6. Unfortunately, experimental data for these values are still missing. 
The PCT diagram measured at $289^{\circ} \mathrm{C}, 317^{\circ} \mathrm{C}$ and $341{ }^{\circ} \mathrm{C}$ for the TiVZrNbHf were reported in [17] and its comparison with the calculated PCT is presented in Figure 3 (f). Differently from the other alloys, the calculated PCT of the TiVZrNbHf alloy presented only a single plateau between $\alpha$ - and $\delta$-phase. The results were the same for all possible configurations of $\theta$ and $r$ used to describe the $\beta$-phase. The calculated PCT predicts a plateau between the $\alpha$-phase with $c_{H} \sim 0.5$ and $\delta$-phase with $c_{H} \sim 0.75$. Above this concentration, the $\mathrm{H}$ concentration of the $\delta$ increases with increase of pressure. D. Karlsson et al. [17] reported the in-situ hydrogenation/dehydrogenation of the TiVZrNbHf using synchrotron XRD measurements. Indeed, no intermediate hydride formation between the $\alpha$ - and $\delta$-phase was observed. The shape of the absorption experimental PCT and the calculated one is quite similar. However, the experimental equilibrium pressures are one order of magnitude higher than the calculated ones, which is probably related to hysteresis. In [17], the authors reported an in-situ experiment in which the fully hydrogenated TiVZrNbHf alloy was subjected to dynamic vacuum $\left(10^{-2}\right.$ atm according to the authors) at $400{ }^{\circ} \mathrm{C}$. In this experiment, the $\delta$-phase phase did not transform back to $\alpha$-phase, however, the volume of the unit cell was reduced indicating a reduction in the $\mathrm{H}$ concentration in the phase. Therefore, we may imagine that the actual $P_{\text {plat }}$ at $400{ }^{\circ} \mathrm{C}$ should be lower than $10^{-2}$ atm and even lower for the lower temperatures in which the PCI measurements were carried out, which is in good agreement with our results. The calculated values of $\Delta H_{\text {plat }}$ and $\Delta S_{\text {plat }}$ are also presented in Table 6.

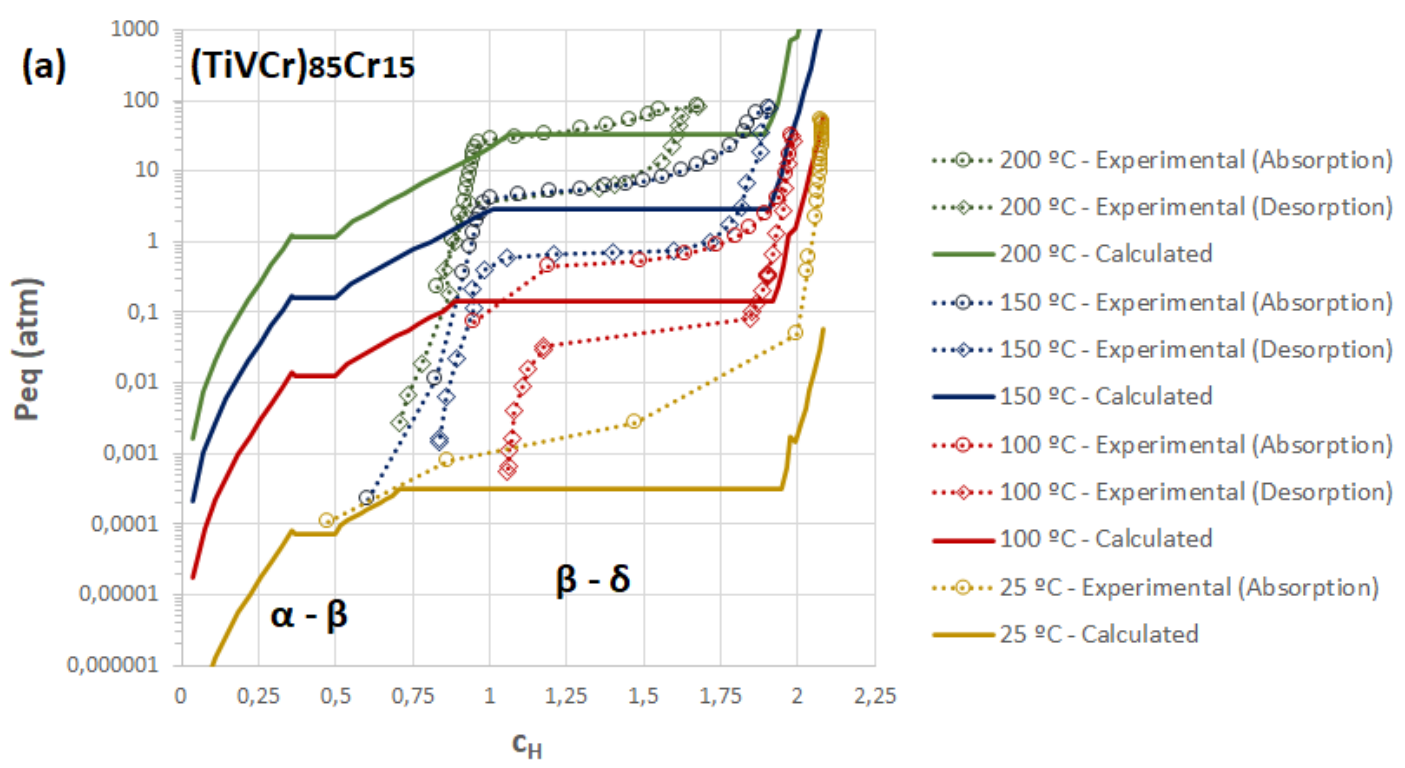




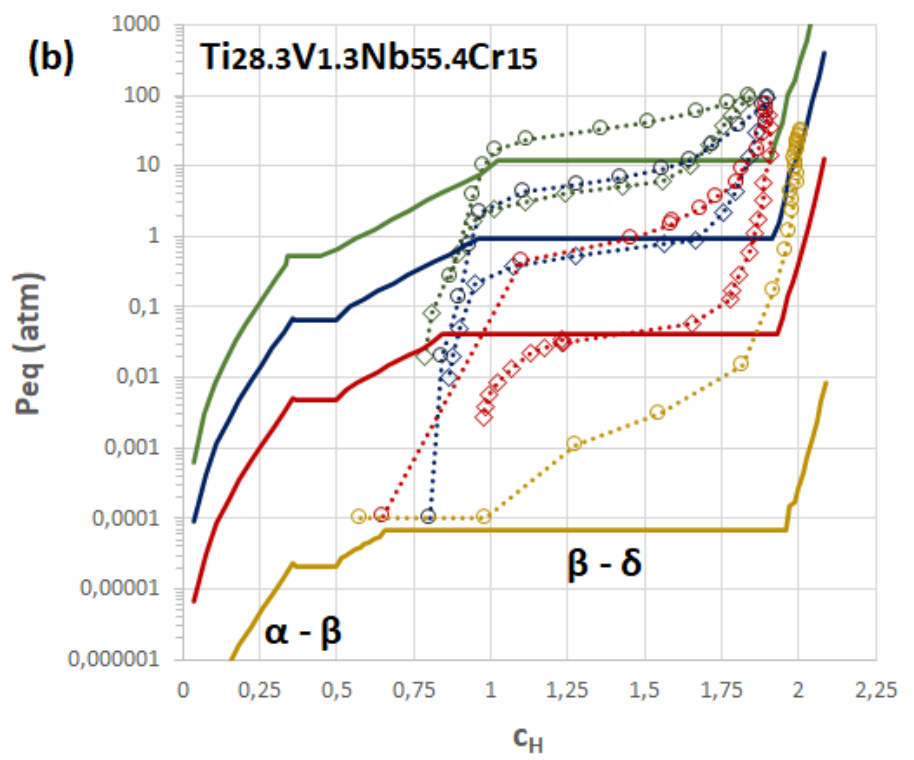

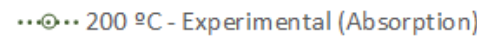

$\cdots \diamond . .200$ ㄷ C - Experimental (Desorption)

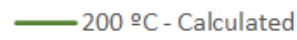

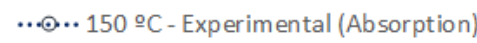

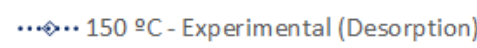

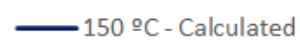

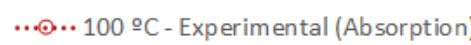

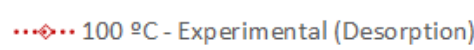

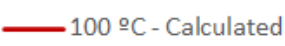

... ... 25 ㄷ - Experimental (Absorption)

-25 ㅇ - Calculated

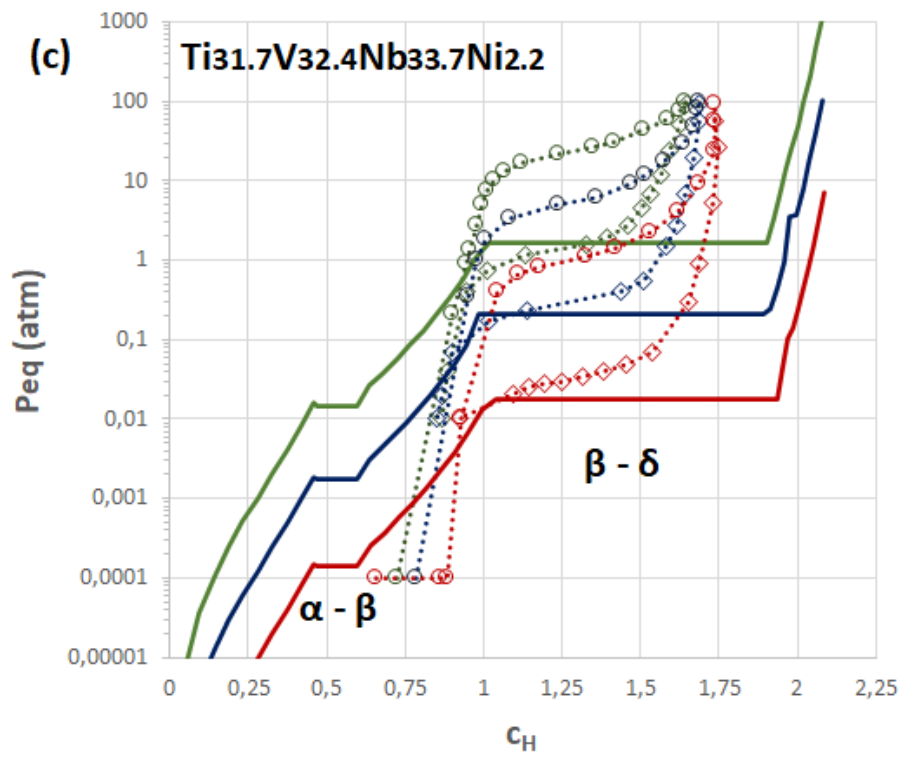

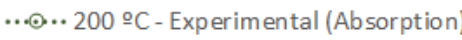
$\cdots \diamond \cdots 200$ 으 - Experimental (Desorption)

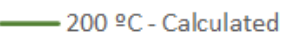

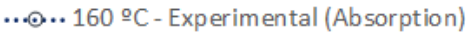
... $\cdots 160$ ㄷ C - Experimental (Desorption) $160 \stackrel{\circ}{\circ}$ - Calculated

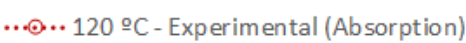
$\cdots \gg \cdot 120 \cong \mathrm{C}-$ Experimental (Desorption)

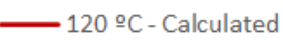

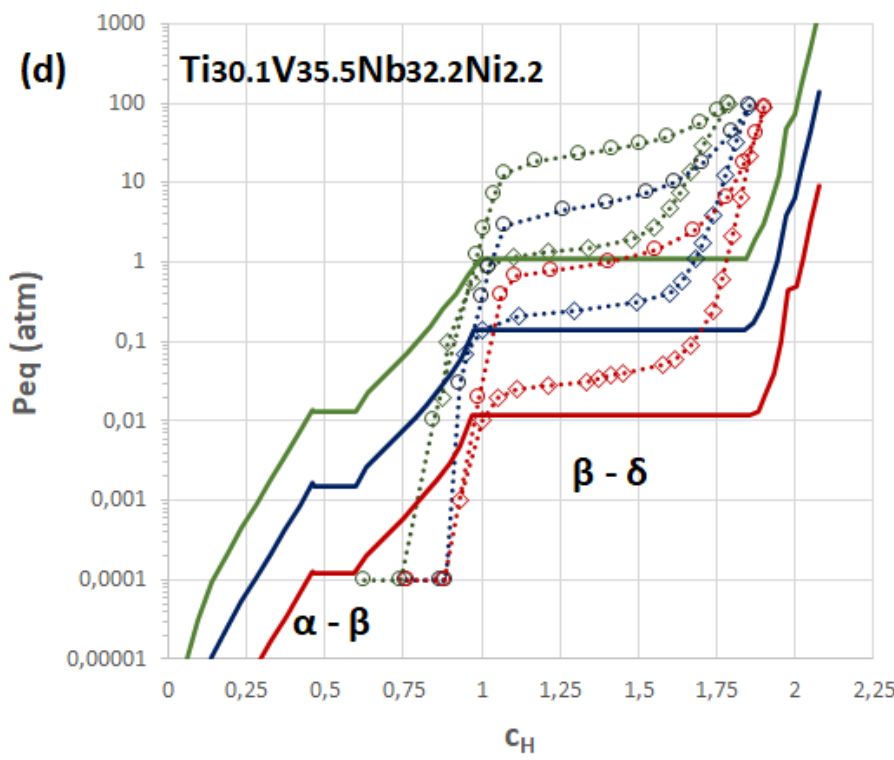

$\cdots \odot \cdots 200$ ㄷ C - Experimental (Absorption)

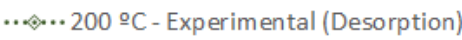
$200 \cong \mathrm{C}$ - Calculated ..๑...160 ㄷ - Experimental (Absorption)

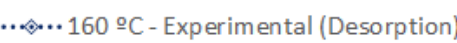

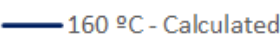

..๑...120 ㄷ - Experimental (Absorption)

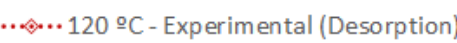

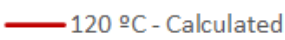



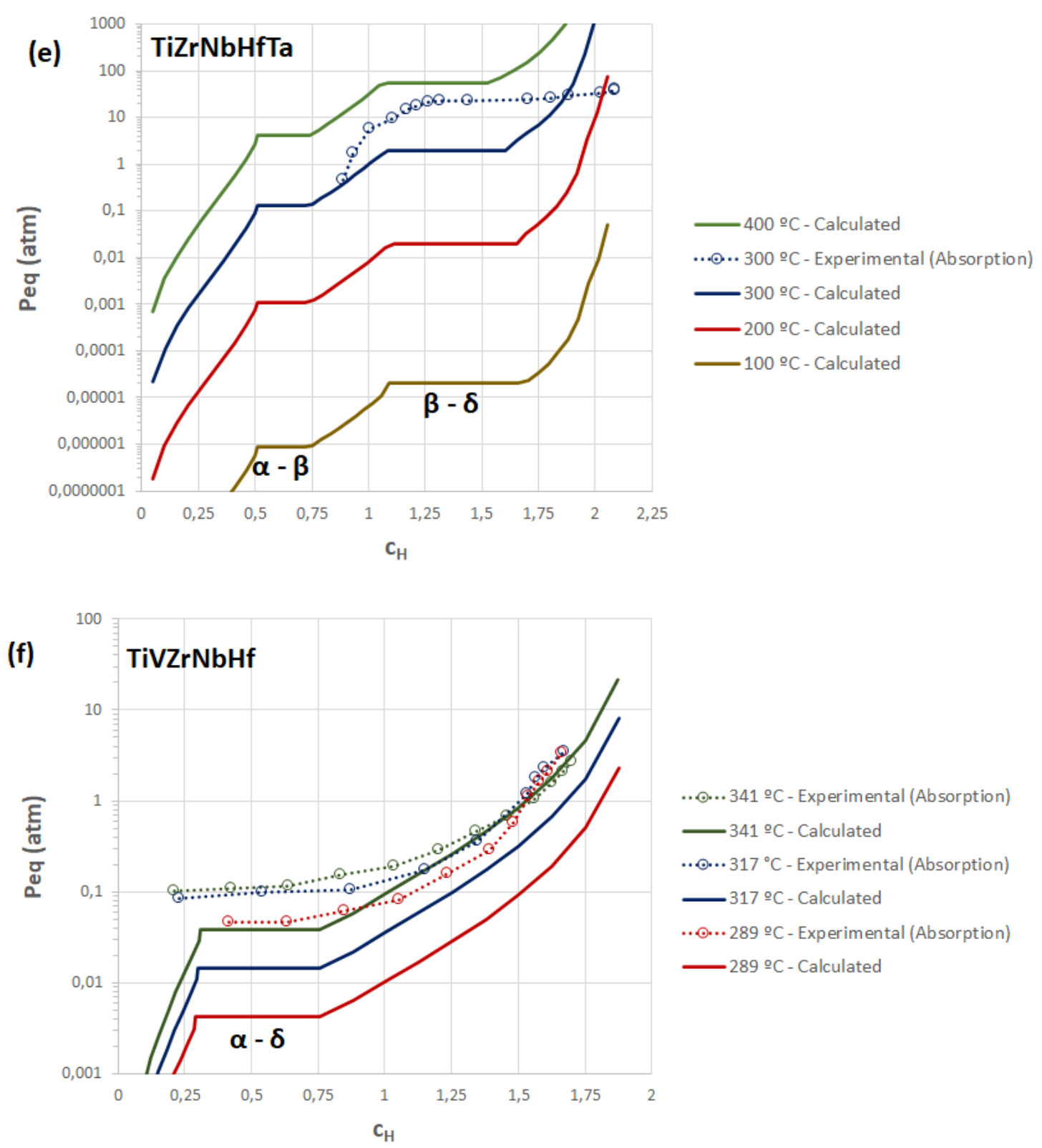

Figure 3: Comparison between calculated and experimental PCT diagrams of (a) $(\mathrm{TiVNb})_{85} \mathrm{Cr}_{15}, \quad$ (b) $\quad \mathrm{Ti}_{28.3} \mathrm{~V}_{1.3} \mathrm{Nb}_{55.4} \mathrm{Cr}_{15}, \quad$ (c) $\quad \mathrm{Ti}_{31.7} \mathrm{~V}_{32.4} \mathrm{Nb}_{33.7} \mathrm{Ni}_{2.2}, \quad$ (d) $\mathrm{Ti}_{30.1} \mathrm{~V}_{35.5} \mathrm{Nb}_{32.2} \mathrm{Ni}_{2.2}$, (e) TiZrNbHfTa (Experimental data taken from [11]), and (f) TiVZrNbHf (Experimental data taken from [17]). 
Table 6: Comparison between the calculated and experimental values of $\Delta H_{\text {plat }}$ and $\Delta S_{\text {plat }} . \Delta H_{\text {plat }}$ and $\Delta S_{\text {plat }}$ are given in $\mathrm{kJ} / \mathrm{mol}$ of $\mathrm{H}$ and $\mathrm{J} / \mathrm{K} . \mathrm{mol}$ of $\mathrm{H}$, respectively.

\begin{tabular}{|c|c|c|c|c|}
\hline \multirow{2}{*}{ Alloy } & \multicolumn{2}{|c|}{ Calculated } & \multicolumn{2}{|c|}{ Experimental } \\
\hline & $\Delta H_{\text {plat }}$ & $\Delta S_{\text {plat }}$ & $\Delta \boldsymbol{H}_{\text {plat }}$ & $\Delta S_{\text {plat }}$ \\
\hline$(\mathrm{TiVNb})_{85} \mathrm{Cr}_{15}$ & $-32.4^{\mathrm{a}} /-34.9^{\mathrm{b}}$ & $-75.2^{\mathrm{a}} /-87.2^{\mathrm{b}}$ & $-32.0^{\mathrm{abs}} /-34.9^{\mathrm{des}}$ & $-83.1^{\mathrm{abs}} /-81.1^{\mathrm{des}}$ \\
\hline $\mathrm{Ti}_{28.3} \mathrm{~V}_{1.3} \mathrm{Nb}_{55.4} \mathrm{Cr}_{15}$ & $-34.1^{\mathrm{a}} /-36.0^{\mathrm{b}}$ & $-74.6^{\mathrm{a}} /-86.5^{\mathrm{b}}$ & $-31.5^{\mathrm{abs}} /-34.4^{\mathrm{des}}$ & $-82.3^{\mathrm{abs}} /-79.3^{\mathrm{des}}$ \\
\hline $\mathrm{Ti}_{31.7} \mathrm{~V}_{32.4 N b 33.7 N i 2.2}$ & $-37.8^{a} /-37.3^{b}$ & $-75.5^{\mathrm{a}} /-81.1^{\mathrm{b}}$ & $-32.0^{\mathrm{abs}} /-35.2^{\mathrm{des}}$ & $-81.8^{\mathrm{abs}} /-76.0^{\mathrm{des}}$ \\
\hline $\mathrm{Ti}_{30.1} \mathrm{~V}_{35.5} \mathrm{Nb}_{32.2} \mathrm{Ni}_{2.2}$ & $-38.2^{\mathrm{a}} /-37.8^{\mathrm{b}}$ & $-74.9^{\mathrm{a}} /-80.4^{\mathrm{b}}$ & $-34.2^{\mathrm{abs}} /-38.1^{\mathrm{des}}$ & $-86.2^{\mathrm{abs}} /-82.4^{\mathrm{des}}$ \\
\hline TiZrNbHfTa & $-44.5^{\mathrm{c}} /-44.1^{\mathrm{d}}$ & $-74.3^{c} /-79.9^{d}$ & - & - \\
\hline TiVZrNbHf & $-51.8^{\mathrm{e}} /-52.5^{\mathrm{f}}$ & $-68.7^{\mathrm{e}} /-72.4^{\mathrm{f}}$ & - & - \\
\hline
\end{tabular}

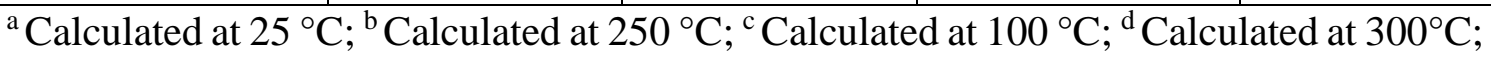
${ }^{\mathrm{e}}$ Calculated at $260{ }^{\circ} \mathrm{C} ;{ }^{\mathrm{f}} \mathrm{Calculated}$ at $360{ }^{\circ} \mathrm{C}$ abs Absorption measurements; ${ }^{\text {des }}$ Desorption measurements.

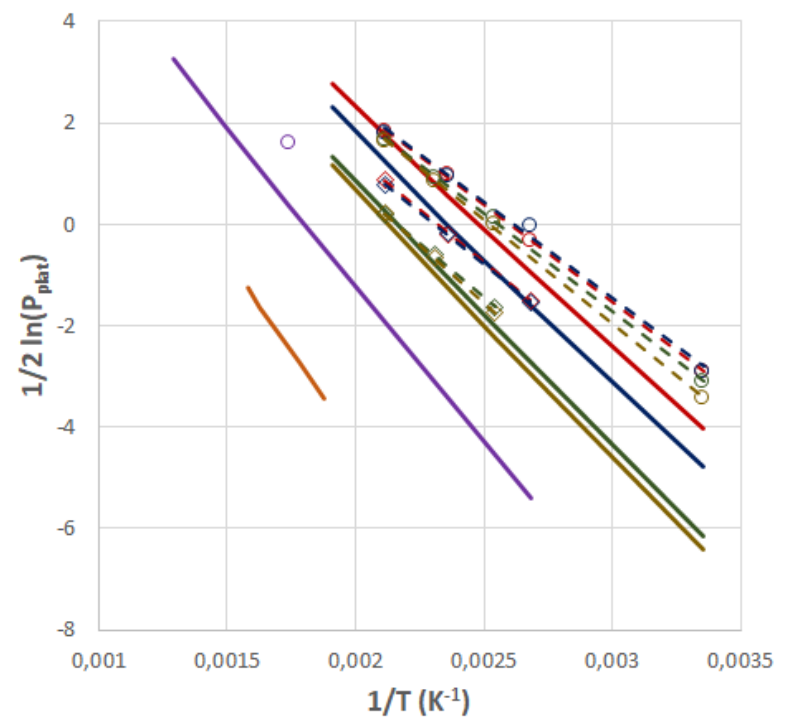

○ (TiVNb)85Cr15 - Experimental (Absorption)

$\diamond($ TiVNb) $85 \mathrm{Cr} 15$ - Experimental (Desorption)

- (TiVNb) $85 \mathrm{Cr} 15$ - Calculated

○ Ti28.1V1.3Nb55.4Cr15 - Experimental (Absorption)

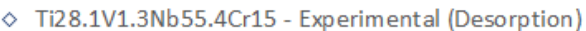

-Ti28.1V1.3Nb55.4Cr15 - Calculated

o Ti31.7V32.4Nb33.7Ni2.2 - Experimental (Absorption)

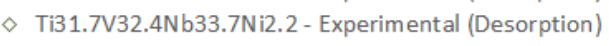

-Ti31.7V32.4Nb33.7Ni2.2 - Calculated

○ Ti30.1V35.5Nb32.2Ni2.2 - Experimental (Absorption)

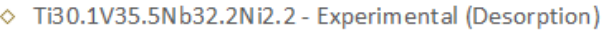

-Ti30.1V35.5Nb32.2Ni2.2 - Calculated

- TiZrNbHfTa - Experimental (Absorption)

- TiZrNbHfTa - Experimental

- TiVZrNbHf - Calculated

Figure 4: Van't Hoff plots comparing experimental and calculated values of plateau pressures. Only for the TiVZrNbHf alloy the plateau pressure between $\alpha$ - and $\delta$-phase is presented. For all others, the pressure of $\alpha-\beta$ plateau is presented. The experimental data for the TiZrNbHfTa alloy was taken from [11]. Since the plateau of the TiVZrNbHf was not clearly observed in the PCT data of [17], the experimental plateau pressure for this alloy is not presented here. 


\section{Discussion}

The good agreement between the calculated and experimental PCTs for the alloys tested here brought to light some important aspects of the thermodynamic of hydrogenmulticomponent alloy systems that is worth to discuss.

Firstly, we can evaluate the role of the configurational entropy of the phases on the PCT calculation. When PE condition is considered, the high ideal configurational entropy of the metal host in multicomponent alloys does not have any impact on the stability of the hydrides as can be seen in equation 16 . The entropy variation caused by the interstitial $\mathrm{H}$ solution is the important entropy term to calculate the PCT. The good agreement between the experimental and calculated values of $\Delta S_{\text {plat }}$ shows that the ideal configurational entropy for interstitial solid solution with SBE proposed by J. Garcés [20] is a suitable model to predict the entropy change during hydrogenation reaction. The common assumption that most entropy change during hydrogenation reaction comes from the vanishing of $\mathrm{H}_{2}$ gas and, therefore, that the entropy difference between the solid phases has a small effect on the plateau pressures is often used [31,32]. Although we agree that the major contribution to $\Delta S_{\text {plat }}$ comes from the conversion of molecular hydrogen gas to dissolved hydrogen, we will show that the difference between the entropies of the solid phases in the plateau is not negligible for PCT calculation. Take as example the (TiVNb) ${ }_{85} \mathrm{Cr}_{15}$, which the calculated $\Delta H_{\text {plat }}$ is $-32.4 \mathrm{~kJ} / \mathrm{mol}$ of $\mathrm{H}$ and $\Delta S_{\text {plat }}$ is $-75.2 \mathrm{~J} / \mathrm{K} . \mathrm{mol}$ of $\mathrm{H}$ for the $\beta-\delta$ plateau at $25^{\circ} \mathrm{C}$, resulting in a $P_{\text {plat }}$ of $3.2 \times 10^{-4} \mathrm{~atm}$. If the difference between the configurational entropies of $\delta$ - and $\beta$-phase was not considered, $\Delta S_{\text {plat }}$ would be $-65.3 \mathrm{~J} / \mathrm{K} . \mathrm{mol}$ of $\mathrm{H}$, resulting in a $P_{\text {plat }}$ of $3.0 \times 10^{-5} \mathrm{~atm}$, i.e., one order of magnitude lower. Such difference would be true for any temperature. Therefore, a good description of the entropy of the solid phases is crucial for PCT calculation aiming at alloy design. Interestingly, the difference between the configurational entropies of the $\mathrm{H}$ interstitial solid solutions seems to be enough to predict the experimental results, which suggests that the difference between the nonconfigurational terms is indeed probably small. Therefore, we believe that a better understating of how the chemical composition affects the $\mathrm{H}$ site occupancy (tetrahedral or octahedral) mainly for the $\beta$-phase is crucial to allows us to select the $\theta$ and $r$ parameters of the configurational entropy model, allowing the application of predictive high-throughput calculations. 
A second point that is worth discussing is the good agreement between the calculated and experimental values of $\Delta H_{\text {plat }}$. In this work, we use the simple approximation of the $\mathrm{H}$ partial molar enthalpy following an ideal mixture law of the alloy's components. The good agreement between the calculated and experimental results shows that at least for the TiVNbCr and TiVNbNi systems in the range of composition studied here, this is a good approximation. However, we believe that this might not be a universal rule. Maybe for other systems, or for other compositions, for example, in the $\mathrm{Cr}$-rich or $\mathrm{Ni}$-rich region of these systems, some deviation from the ideal mixture law might be important. Even though, the parametrization of the $h_{M}$ term of equation 17 can be further developed using either ab initio calculation or experimental data. The ideal mixture law was only the first choice to test the proposed model.

Finally, in our approach the SBE was considered only in the entropy and not in the enthalpy formulation. If the strain caused by the $\mathrm{H}$ interstitial solid solution in the host metal lattice and repulsive $\mathrm{H}-\mathrm{H}$ interactions were considered in the enthalpy formulation, the $\mathrm{H}$ partial molar enthalpy of a phase would not be constant, but it would depend on the H concentration. Indeed, since the classical work of R. Lacher in 1935 [33], many authors have used the approach of describing the $\mathrm{H}$ partial molar enthalpy as a linear function of the hydrogen concentration [34-36]. In our approach, we consider that when such increase of energy caused by $\mathrm{H}-\mathrm{H}$ interactions or lattice strain manifests, the system responds by organizing the $\mathrm{H}$ atoms in a new ordered phase that eliminate this excess energy. Therefore, the new phase with lower configurational entropy (mathematically expressed by the lower $\theta$ parameter) is formed. Since in this work we use the same $h_{M}$ value to describe the enthalpies of the $\alpha$-phase and $\beta$-phase, the first plateau between these two phases is only a result of their different configurational entropies. For the case where the $\beta$-phase continues to be $\mathrm{BCC}$ with $\mathrm{H}$ atoms at specific tetrahedral sites, the spinodal decomposition could be described only by the different configurational entropies of the two phases. In this case, the lattice strain and $\mathrm{H}-\mathrm{H}$ interactions would manifest on the SBE and it would be account into the $r$ term of the configurational entropy equation. Determining stress/strain field and local H-H interaction energy is rather complicated. Therefore, the possibility of calculating reasonably accurate PCTs without the need of these quantities makes the model much more useful for alloy design and high-throughput calculations. Therefore, we reinforce that a deeper evaluation of the $\mathrm{H}$ ordering in the 
interstitial sites of multicomponent alloys is of fundamental importance to improve the predictability of PCT calculations.

\section{Conclusions}

In this work we presented a thermodynamic model to calculate PCT diagrams. The configurational entropy of the phases was described using the equation formulated by $\mathrm{J}$. Garcés for interstitial solid solution with site blocking effect. The enthalpy of $\mathrm{H}$ mixing in the possible phases was considered to vary linearly with the $\mathrm{H}$ concentration, meaning a constant $\mathrm{H}$ partial molar enthalpy. For multicomponent phases, the partial molar enthalpy was approximated by an ideal mixture law of the alloy's components, and the values for the pure elements were parametrized either by experimental data or DFT calculation. The model was applied to six BCC multicomponent alloys and the calculated PCTs were compared against the experimental ones. The good agreement between the calculated and experimental results shows that this model can be used as a basis for calculating reasonably accurate PCTs and become a powerful tool for alloy design and high-throughput calculations.

\section{Acknowledgements}

This work was financed in part by the Serrapilheira Institute (grant number Serra1709-17362) and in part by the CAPES-COFECUB cooperation program (CAPES project numbers: 88887.387428/2019-00 and 88887.191910/2018-00, COFECUB project number: Ph-C951/19). This study was also financed in part by the Coordenação de Aperfeiçoamento de Pessoal de Nível Superior - Brasil (CAPES) - Finance Code 001, Federal University of Sao Carlos, Graduate Program in Materials Science and Engineering.

\section{References}

[1] M. Marinelli, M. Santarelli, Hydrogen storage alloys for stationary applications, J. Energy Storage. 32 (2020) 101864. doi:10.1016/j.est.2020.101864.

[2] K. Manickam, P. Mistry, G. Walker, D. Grant, C.E. Buckley, T.D. Humphries, M. Paskevicius, T. Jensen, R. Albert, K. Peinecke, M. Felderhoff, Future perspectives of thermal energy storage with metal hydrides, Int. J. Hydrogen 
Energy. 44 (2019) 7738-7745. doi:10.1016/j.ijhydene.2018.12.011.

[3] J. Bellosta von Colbe, J.R. Ares, J. Barale, M. Baricco, C. Buckley, G. Capurso, N. Gallandat, D.M. Grant, M.N. Guzik, I. Jacob, E.H. Jensen, T. Jensen, J. Jepsen, T. Klassen, M. V. Lototskyy, K. Manickam, A. Montone, J. Puszkiel, S. Sartori, D.A. Sheppard, A. Stuart, G. Walker, C.J. Webb, H. Yang, V. Yartys, A. Züttel, M. Dornheim, Application of hydrides in hydrogen storage and compression: Achievements, outlook and perspectives, Int. J. Hydrogen Energy. 44 (2019) 7780-7808. doi:10.1016/j.ijhydene.2019.01.104.

[4] Q. Cheng, D. Sun, X. Yu, Metal hydrides for lithium-ion battery application: A review, J. Alloys Compd. 769 (2018) 167-185. doi:10.1016/j.jallcom.2018.07.320.

[5] M. V. Lototskyy, I. Tolj, L. Pickering, C. Sita, F. Barbir, V. Yartys, The use of metal hydrides in fuel cell applications, Prog. Nat. Sci. Mater. Int. 27 (2017) 320. doi:10.1016/j.pnsc.2017.01.008.

[6] L. Ouyang, J. Huang, H. Wang, J. Liu, M. Zhu, Progress of hydrogen storage alloys for Ni-MH rechargeable power batteries in electric vehicles: A review, Mater. Chem. Phys. 200 (2017) 164-178. doi:10.1016/j.matchemphys.2017.07.002.

[7] D.B. Miracle, High entropy alloys as a bold step forward in alloy development, Nat. Commun. (2019) 1-3. doi:10.1038/s41467-019-09700-1.

[8] D.B. Miracle, O.N. Senkov, A critical review of high entropy alloys and related concepts, Acta Mater. 122 (2017) 448-511. doi:10.1016/j.actamat.2016.08.081.

[9] M. Sahlberg, D. Karlsson, C. Zlotea, U. Jansson, Superior hydrogen storage in high entropy alloys, Sci. Rep. 6 (2016) 36770. doi:10.1038/srep36770.

[10] V. Pacheco, G. Lindwall, D. Karlsson, J. Cedervall, S. Fritze, G. Ek, P. Berastegui, M. Sahlberg, U. Jansson, Thermal Stability of the HfNbTiVZr HighEntropy Alloy, Inorg. Chem. 58 (2018) 811-820. doi:10.1021/acs.inorgchem.8b02957.

[11] C. Zlotea, M.A. Sow, G. Ek, J.-P. Couzinié, L. Perrière, I. Guillot, J. Bourgon, K.T. Møller, T.R. Jensen, E. Akiba, M. Sahlberg, Hydrogen sorption in TiZrNbHfTa high entropy alloy, J. Alloys Compd. 775 (2018) 667-674. doi:10.1016/j.jallcom.2018.10.108.

[12] M.M. Nygård, G. Ek, D. Karlsson, M.H. Sørby, M. Sahlberg, B.C. Hauback, Counting electrons - A new approach to tailor the hydrogen sorption properties of high-entropy alloys, Acta Mater. 175 (2019) 121-129. doi:10.1016/j.actamat.2019.06.002.

[13] F. Marques, H.C. Pinto, S.J.A. Figueroa, F. Winkelmann, M. Felderhoff, W.J. Botta, G. Zepon, Mg-containing multi-principal element alloys for hydrogen storage: A study of the MgTiNbCr0.5Mn0.5Ni0.5 and Mg0.68TiNbNi0.55 compositions, Int. J. Hydrogen Energy. 45 (2020) 19539-19552. doi:10.1016/j.ijhydene.2020.05.069.

[14] J. Montero, G. Ek, L. Laversenne, V. Nassif, G. Zepon, M. Sahlberg, C. Zlotea, Hydrogen storage properties of the refractory $\mathrm{Ti}$ e $\mathrm{V}$ e $\mathrm{Zr}$ e $\mathrm{Nb}$ e Ta multi- 
principal element alloy, J. Alloys Compd. 835 (2020) 155376. doi:10.1016/j.jallcom.2020.155376.

[15] J. Montero, C. Zlotea, G. Ek, J. Crivello, L. Laversenne, M. Sahlberg, TiVZrNb Multi-Principal-Element Alloy: Synthesis Optimization, Structural, and Hydrogen Sorption Properties, Molecules. 24 (2019) 2799. doi:10.3390/molecules24152799.

[16] G. Zepon, D.R. Leiva, R.B. Strozi, A. Bedoch, S.J.A. Figueroa, T.T. Ishikawa, W.J. Botta, Hydrogen-induced phase transition of $\mathrm{MgZrTiFe} 0.5 \mathrm{Co} 0.5 \mathrm{Ni} 0.5$ high entropy alloy, Int. J. Hydrogen Energy. 43 (2018) 1702-1708. doi:10.1016/j.ijhydene.2017.11.106.

[17] D. Karlsson, G. Ek, J. Cedervall, C. Zlotea, K.T. Møller, T.C. Hansen, J. Bednarčík, M. Paskevicius, M.H. Sørby, T.R. Jensen, U. Jansson, M. Sahlberg, Structure and Hydrogenation Properties of a HfNbTiVZr High-Entropy Alloy, Inorg. Chem. 57 (2018) 2103-2110. doi:10.1021/acs.inorgchem.7b03004.

[18] T.B. Flanagan, W.A. Oates, Some thermodynamic aspects of metal hydrogen systems, J. Alloys Compd. 404-406 (2005) 16-23. doi:10.1016/j.jallcom.2004.11.108.

[19] D.R. Burgess Jr, Thermochemical Data, in: P.J. Linstrom, W.G. Mallard (Eds.), NIST Chem. WebBook, NIST Stand. Ref. Database Number 69, n.d. doi:https://doi.org/10.18434/T4D303.

[20] J. Garcés, The configurational entropy of mixing of interstitials solid solutions, Appl. Phys. Lett. 96 (2010) 67-70. doi:10.1063/1.3400221.

[21] Y. Fukai, The Metal-Hydrogen System, Springer Berlin Heidelberg, Berlin, Heidelberg, 2005. doi:10.1007/3-540-28883-X.

[22] T.B. Flanagan, W.A. Oates, Thermodynamics of intermetallic CompoundHydrogen Systems, in: 1988: pp. 49-85. doi:10.1007/3540183337_10.

[23] T. Schober, H. Wenzl, The systems NbH(D), TaH(D), VH(D) : Structures, phase diagrams, morphologies, methods of preparation, in: G. Alefeld, J. Völkl (Eds.), Springer Berlin Heidelberg, Berlin, Heidelberg, 1978: pp. 11-71. doi:10.1007/3540-08883-0_18.

[24] A.G. Aleksanyan, D.G. Mayilyan, S.K. Dolukhanyan, V.S. Shekhtman, O.P. TerGalstyan, Formation of triple alloys and their hydrides in the Ti-Zr-Hf-H system, Int. J. Self-Propagating High-Temperature Synth. 19 (2010) 34-39. doi:10.3103/s1061386210010061.

[25] S.K. Dolukhanyan, A.G. Aleksanyan, O.P. Ter-Galstyan, V.S. Shekhtman, M.K. Sakharov, G.E. Abrosimova, Specifics of the formation of alloys and their hydrides in Ti-Zr-H system, Russ. J. Phys. Chem. B. 1 (2007) 563-569. doi:10.1134/s1990793107060085.

[26] V.S. Shekhtman, S.K. Dolukhanyan, A.G. Aleksanyan, D.G. Mayilyan, O.P. TerGalstyan, M.K. Sakharov, S.S. Khasanov, Effect of hydrogen on the structure of alloys formed in the Ti-Zr-Hf-H system, Int. J. Self-Propagating HighTemperature Synth. 19 (2010) 40-48. doi:10.3103/s1061386210010073.

[27] P. Blaha, K. Schwarz, G.K.. Madsen, D. Kvasnicka, J. Luitz, WIEN2k, An 
Augmented Plane Wave + Local Orbitals Program for Calculating Crystal Properties (Karlheinz Schwarz, Techn. Universität Wien, Austria), (2001). doi:ISBN: 3950103112.

[28] P. Blaha, K. Schwarz, F. Tran, R. Laskowski, G.K.H. Madsen, L.D. Marks, WIEN2k: An APW+lo program for calculating the properties of solids, J. Chem. Phys. 152 (2020). doi:10.1063/1.5143061.

[29] J.P. Perdew, K. Burke, M. Ernzerhof, Generalized Gradient Approximation Made Simple, Phys. Rev. Lett. 77 (1996) 3865-3868. doi:10.1103/PhysRevLett.77.3865.

[30] R. Griessen, T. Riesterer, Heat of formation models, in: Hydrog. Intermet. Compd. I, 1988: pp. 219-284. doi:10.1007/3540183337_13.

[31] K.H.J. Buschow, P.C.P. Bouten, A.R. Miedema, Hydrides formed from intermetallic compounds of two transition metals: A special class of ternary alloys, Reports Prog. Phys. 45 (1982) 937-1039. doi:10.1088/00344885/45/9/001.

[32] A. Züttel, Materials for hydrogen storage, Mater. Today. 6 (2003) 24-33. doi:10.1016/S1369-7021(03)00922-2.

[33] J.R. Lacher, A theoretical formula for the solubility of hydrogen in palladium, Proc. R. Soc. London. Ser. A - Math. Phys. Sci. 161 (1937) 525-545. doi:10.1098/rspa.1937.0160.

[34] H.A. Kierstead, A theory of multiplateau hydrogen absorption isotherms, J. LessCommon Met. 71 (1980) 303-309. doi:10.1016/0022-5088(80)90213-1.

[35] H.A. Kierstead, Enhancement of the Lacher and Rees theories of hydrogen absorption isotherms, J. Less-Common Met. 77 (1981) 281-285. doi:10.1016/0022-5088(81)90180-6.

[36] M.V. Lototsky, V.A. Yartys, V.S. Marinin, N.M. Lototsky, Modelling of phase equilibria in metal-hydrogen systems, J. Alloys Compd. 356-357 (2003) 27-31. doi:10.1016/S0925-8388(03)00095-1. 